\title{
B-box containing protein 1 from Malus domestica (MdBBX1) is involved in the abiotic stress response
}

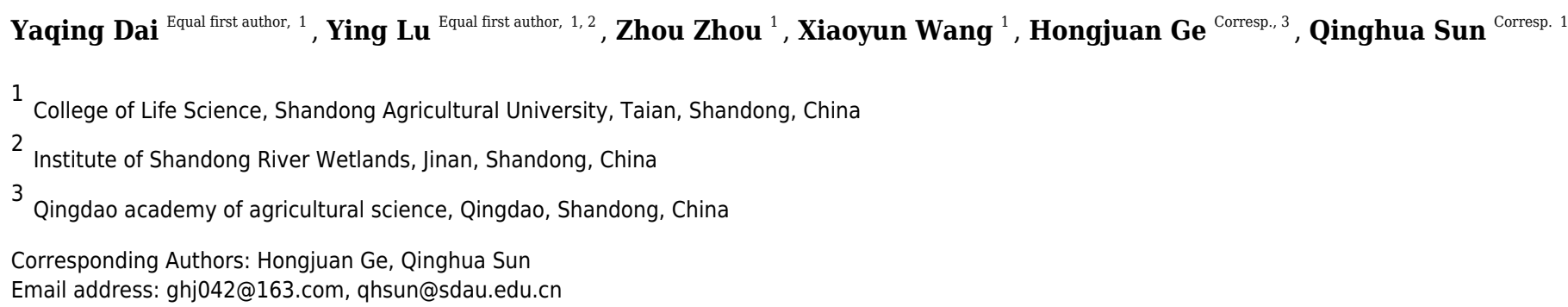

B-box proteins (BBXs), which act as transcription factors, mainly regulate photomorphogenesis. However, the molecular functions underlying the activity of plant BBXs in response to abiotic stress remain largely unclear. In this investigation, we found that a BBX from Malus domestica (MdBBX1) was involved in the response to various abiotic stresses. The expression of $M d B B X 1$ was significantly upregulated in response to abiotic stresses and abscisic acid (ABA). Recombinant MdBBX1 increased stress tolerance in Escherichia coli cells. In addition, overexpression of MdBBXI in Arabidopsis decreased sensitivity to exogenous $A B A$, resulting in a germination rate and root length that were greater and longer, respectively, than those of wild-type (WT) plants. Moreover, the expression of $A B I 5$ was decreased in MdBBX1-overexpressing lines under ABA treatment. After salt and drought treatments, compared with the WT plants, the MdBBXI transgenic plants displayed enhanced tolerance and had a higher survival rate. Furthermore, under salt stress, increased proline (PRO) contents, decreased levels of malondialdehyde (MDA), increased activity of antioxidant enzymes (superoxide dismutase [SOD], peroxidase [POD], catalase [CAT] and ascorbate peroxidase [APX]) and decreased accumulation of reactive oxygen species (ROS) were observed in the MdBBX1-overexpressing plants. Overall, our results provide evidence that $M d B B X 1$ might play a critical role in the regulation of abiotic stress tolerance by reducing the generation of ROS. 


\section{B-box containing protein 1 from Malus domestica (MdBBX1) \\ 2 is involved in the abiotic stress response}

3

4 Yaqing Dai1 ${ }^{1 \S}$, Ying Lu ${ }^{1,2 \S}$, Zhou Zhou ${ }^{1}$, Xiaoyun Wang ${ }^{1}$, Hongjuan $\mathrm{Ge}^{3 *}$ and Qinghua Sun ${ }^{*}$

${ }^{1}$ College of Life Science, State Key Laboratory of Crop Biology, Shandong Agricultural University, Taian, Shandong,

$7 \quad 271018$, PR China

$8 \quad{ }^{2}$ Institute of Shandong River Wetlands, Jinan, Shandong, 271100, PR China

$9{ }^{3}$ Qingdao Academy of Agricultural Science, Qingdao, Shandong, 266000, PR China

$11 \S$ These authors contributed equally to this article

12

13 Corresponding author:

14 Qinghua Sun ${ }^{1}$,

15 Email address: qhsun@sdau.edu.cn

16 Hongjuan $\mathrm{Ge}^{3}$,

17 Email address: ghj042@163.com

18

19

20

21 


\section{Abstract}

23 B-box proteins (BBXs), which act as transcription factors, mainly regulate photomorphogenesis.

24 However, the molecular functions underlying the activity of plant BBXs in response to abiotic

25 stress remain largely unclear. In this investigation, we found that a BBX from Malus domestica

$26(M d B B X 1)$ was involved in the response to various abiotic stresses. The expression of $M d B B X 1$

27 was significantly upregulated in response to abiotic stresses and abscisic acid (ABA). Recombinant

28 MdBBX1 increased stress tolerance in Escherichia coli cells. In addition, overexpression of

$29 M d B B X 1$ in Arabidopsis decreased sensitivity to exogenous ABA, resulting in a germination rate

30 and root length that were greater and longer, respectively, than those of wild-type (WT) plants.

31 Moreover, the expression of $A B I 5$ was decreased in $M d B B X 1$-overexpressing lines under ABA

32 treatment. After salt and drought treatments, compared with the WT plants, the $M d B B X 1$

33 transgenic plants displayed enhanced tolerance and had a higher survival rate. Furthermore, under

34 salt stress, increased proline (PRO) contents, decreased levels of malondialdehyde (MDA),

35 increased activity of antioxidant enzymes (superoxide dismutase [SOD], peroxidase [POD],

36 catalase $[\mathrm{CAT}]$ and ascorbate peroxidase $[\mathrm{APX}])$ and decreased accumulation of reactive oxygen

37 species (ROS) were observed in the $M d B B X 1$-overexpressing plants. Overall, our results provide

38 evidence that $M d B B X 1$ might play a critical role in the regulation of abiotic stress tolerance by

39 reducing the generation of ROS. 


\section{Introduction}

42 B-box containing proteins (BBXs) are typical zinc finger transcription factors with 1 or 2 zinc-

43 binding B-box domain(s) at the N-terminus of protein sequence and occasionally with a CCT

44 domain (for CONSTANS, CONSTANS-like, TOC1) at the C-terminus (Gangappa \& Botto, 2014;

45 Khanna et al., 2009). There are 32 family members of BBX in Arabidopsis thaliana and they are

46 divided into five subfamilies according to their amino acid sequences. BBXs in group I-III contain

47 a CCT domain that participates in the regulation of the transcription or nuclear import. Groups I,

48 II, and IV contain two B-box motifs, while groups III and V harbor only one B-box motif

49 (Gangappa \& Botto, 2014; Khanna et al., 2009). BBX members in different groups have been

50 identified to function in regulating anthocyanin accumulation, flowering, shade avoidance and

51 photomorphogenesis, as well as responses to stress (An et al., 2019; An et al., 2020; Chang et al.,

52 2008; Crocco et al., 2011; Crocco et al., 2015; Datta et al., 2007; Datta et al., 2008; Fang et al.,

53 2019; Gangappa et al., 2013; Heng et al., 2019a; Heng et al., 2019b; Sarmiento, 2013; Wang et

54 al., 2013; Wei et al., 2016; Yadav et al., 2019; Zhang et al., 2017).

55 In the BBX family of Arabidopsis, the members in the same group may have different

56 functions. For example, both BBX21 and BBX24 belong to the same structural group, group IV

57 (whose members have two B-box motifs and no CCT domain), but their functions in regulating

58 photomorphogenesis are opposite. BBX21 is a positive regulator of photomorphogenesis, whereas

59 BBX24 is a negative regulator (Xu et al., 2016). Despite the opposite functions of BBX21 and

60 BBX24, the antagonistic modulating ability of both depends on HY5 (Job et al., 2018), which is a 
61 central downstream regulator of light-mediated developmental processes and can bind to the

62 promoter of the $A B I 5$ gene to activate its expression (Chen et al., 2008). To illustrate the molecular

63 mechanism for underlying the contrasting functions of BBX21 and BBX24 (Job et al., 2018), the

64 protein sequences of these two genes were compared, and the results revealed that their functional

65 differences were mainly determined by different sequences of the C-terminal region. In support of

66 this notion, the researchers constructed two vectors that expressed BBX24 and BBX21 proteins

67 fused to each other's C-terminal sequences; the fusion proteins were subsequently named "BB24C21" and "BB21C24", respectively. The results showed that, similar to the overexpression of BBX21, the overexpression of BB24C21 could transcriptionally upregulate the expression of HY5. Whereas over-expression of BB21C24 did not have any effect on the mRNA levels of HY5

71 (Job et al., 2018). Furthermore, the researchers found that BBX21 could mediate HY5 post transcriptionally. In contrast, BBX24 could prevent HY5 from binding to the promoter of the target gene, probably by heterodimerizing with HY5 and inhibiting its ability to bind to DNA. In conclusion, closely related BBXs may perform opposite functions. salt tolerance-related protein, BBX24 can negatively regulate the expression of many stress-related genes (Nagaoka \& Takano, 2003). AtBBX24 transgenic plants were shown to be more salt tolerant than wild-type (WT) plants under salt stress. BBX5, a group I member, contains two B-box 
81 resistance to abiotic stresses (Min et al., 2015). Overexpression of a BBX protein in banana

82 obviously improved its tolerance to biotic and abiotic stresses, such as pathogen infection and

83 chilling (Chen et al., 2012). Similarly, overexpression of OsBBX25 in Arabidopsis thaliana can

84 increase plant tolerance to abiotic stresses (Liu et al., 2012). Heterologous expression of $A t B B X 21$

85 enhances the photosynthesis rate and alleviates photoinhibition in Solanum tuberosum (Crocco et

86 al., 2018). In addition, some tomato $B B X$ genes can also be induced in response to heat, drought

87 and phytohormones (Chu et al., 2016). Overall, BBX proteins play vital roles in regulating various

88 stress responses.

89 Our previous study reported that there are $64 B B X s$ in the apple genome, which can be divided

90 into five groups, similar to the Arabidopsis BBX family. Some $M d B B X$ genes are induced in

91 response to different abiotic stresses, indicating that $M d B B X s$ may participate in abiotic stress

92 responses (Liu et al., 2018). A recent study found that MdBBX10 from apple could promote

93 tolerance to drought and salt stresses in Arabidopsis (Liu et al., 2019). MdBBX10 belongs to group

$94 \mathrm{~V}$, and contains one B-box domain, but no CCT domain. Overexpression of MdBBX10 in

95 Arabidopsis dramatically improved the tolerance to abiotic stress and increased sensitivity to ABA

96 during the seed germination and seedling stages (Liu et al., 2019). Here, we demonstrated that a

97 BBX member of group I, MdBBX1, which contains two B-box domains and a CCT domain (Fig.

$98 \mathrm{~S} 1$ ), also responds to abiotic stress, but causes insensitivity to exogenous ABA when

99 overexpression in Arabidopsis, which is opposite to the response of MdBBX10 to ABA. 


\section{Materials \& Methods}

\section{Plant growth conditions and treatments}

102 For organ-specific expression analyses, different apple organs, including roots, stems, leaves,

103 flowers and fruits, were sampled from six-year-old apple trees growing at the experimental station

104 of Shandong Agricultural University (Tai'an, Shandong, China).

105 Apple (golden delicious) seedlings were cultivated under greenhouse conditions (relative

106 humidity of $60-75 \%$ ) at $22 \pm 1{ }^{\circ} \mathrm{C}$ with a $16 \mathrm{~h} \mathrm{light} / 8 \mathrm{~h}$ dark photoperiod for approximately one

107 year. Then, the uniformly growing seedlings were selected for stress treatments. For salt and

108 drought stress treatments, the apple seedlings were watered with solutions of $250 \mathrm{mM} \mathrm{NaCl}$ or

$10925 \%(\mathrm{w} / \mathrm{v})$ polyethylene glycol-6000 (PEG-6000), and control seedling received the same amount

110 of water only. For ABA treatment, $100 \mu \mathrm{M}$ ABA solutions were directly sprayed on the seedlings.

111 For cold stress, the apple seedlings were subjected to $4^{\circ} \mathrm{C}$ condition, while seedlings growing at

112 room temperature $\left(25^{\circ} \mathrm{C}\right)$ were used as the controls. Samples were collected from 3 different kinds

113 of seedlings at $0,3,6,9$ and $12 \mathrm{~h}$ after treatment, as was done in a previous study (Yuan et al.,

114 2013). Then, the collected samples were immediately frozen in liquid nitrogen and stored at $-70^{\circ} \mathrm{C}$

115 till used. Subsequently, the total RNA was extracted from the collected samples using an improved

116 cetyl-trimethylammonium bromide (CTAB) procedure (Gasic et al., 2004).

117 The seeds of WT (Col-0) and transgenic Arabidopsis were disinfected and sown on 1/2

118 Murashige and Skoog (MS) media. After culturing at $4^{\circ} \mathrm{C}$ for 3 days to undergo vernalization, the 
119 seedlings were transferred to a greenhouse condition, which included a $22 \pm 1{ }^{\circ} \mathrm{C}$ temperature with

120 a $16 \mathrm{~h}$ light/8 h photoperiod. Then, 3-week-old WT and transgenic plants were treated with 250

$121 \mathrm{mM} \mathrm{NaCl}$ or 25\% (w/v) PEG-6000 as described by Liu et al., (2019), and the control seedlings

122 were treated with water only. Plant growth status was observed and the survival rates were

123 determined daily. Each treatment was performed at least three times.

124 Quantitative real-time PCR (qRT-PCR) analysis

125 QRT-PCR is a commonly used approach for the quantitative detection of gene expression in real

126 time (Deepak et al., 2007). All the primers used in this investigation were designed according to

127 the target gene sequences via the Beacon Designers software and were shown in Table S1. qRT-

128 PCR was carried out using a SYBR ${ }^{\circledR}$ PrimeScript ${ }^{\text {TM }}$ RT-PCR Kit (TaKaRa, Dalian, China) and

129 run on a CFX96TM Real-Time PCR Detection System (Bio-Rad, Hercules, CA, USA). The

130 Arabidopsis Actin8 and apple actin genes were used as reference genes (Wang et al., 2016). The

131 thermal cycling parameters were as follows: 40 cycles of $95^{\circ} \mathrm{C}$ denaturation for $15 \mathrm{~s}, 55^{\circ} \mathrm{C}$

132 annealing for $20 \mathrm{~s}$ and $70^{\circ} \mathrm{C}$ extension for $15 \mathrm{~s}$. The qRT-PCR data were analyzed by the $2^{-\Delta \Delta \mathrm{Ct}}$

133 method (Livak \& Schmittgen, 2001). The relative expression of MdBBX1 in the treated samples

134 was compared with that in the nontreated samples at each treatment time point with significant

135 differences $(\mathrm{P}<0.05)$ determined based on Tukey's multiple test.

\section{Subcellular localization of $\boldsymbol{M d B B X 1}$}

137 The full-length coding sequence of $M d B B X 1$ was amplified from apple and inserted into the

138 pROKII vector containing a GFP gene and the CaMV35S promoter. Cells of Agrobacterium 
139 tumefaciens GV3101 with the recombinant plasmid were cultured overnight, resuspended in

140 osmotic solution (10 mM $\mathrm{MgCl}_{2}, 10 \mathrm{mM}$ 2-[N-morpholino] ethanesulfonic acid [MES] and 150

$141 \mathrm{mM}$ acetosyringone), and then injected into the leaves of 1-month-old Nicotiana benthamiana

142 plants. The fluorescent signal of $M d B B X 1$-GFP was detected via a confocal microscope (LSM 510

143 META, Carl Zeiss) after 2-3 days. The nuclei were subsequently stained with $100 \mathrm{~g} / \mathrm{ml} \mathrm{4',6} \mathrm{-}$

144 diamidino-2-phenylindole (DAPI) (Solarbio, Beijing, China) for $10 \mathrm{~min}$. Leaves overexpressing

145 35S-GFP were used as controls (Wang et al., 2018).

\section{Construction of expression plasmids}

147 The cDNA sequence of $M d B B X 1$ was inserted into the polyclonal sites of pET-30a (+) (Novagen),

148 which contained His-tagged sequences. Then, the recombinant vector was transformed into

149 Escherichia coli BL21 cells. The recombinant sequences in the plasmids were sequenced by

150 Sangon Biotechnology Company (Shanghai, China).

\section{Survival test of Escherichia under different abiotic stresses}

152 Survival analysis of Escherichia coli under salt and drought stress was conducted as described by

153 Du et al., (2014). The cells were cultured in Luria-Bertani (LB) liquid media until the OD 600

154 reached $0.4-0.6$, and then the expression of the recombinant protein was induced for $2 \mathrm{~h}$ using

155 isopropyl $\beta$-D-1-thiogalactopyranoside (IPTG) at $37^{\circ} \mathrm{C}$. All the bacterial cultures were first diluted

156 to an $\mathrm{OD}_{600}$ of 0.6 and then diluted $10^{-3}, 10^{-4}$ and $10^{-5}$ times. For the survival test on solid media,

$15710 \mu \mathrm{L}$ cultures of each dilution were spotted onto solid LB media that included $500 \mathrm{mM} \mathrm{KCl,} 500$

$158 \mathrm{mM} \mathrm{NaCl}$ or $600 \mathrm{mM}$ mannitol and incubated for $12 \mathrm{~h}$ at $37^{\circ} \mathrm{C}$. Then, the colony numbers in each 
159 dish for the culture diluted to $10^{-5}$ were counted. Each experiment was performed at least three

160 times.

161 For the survival test in liquid media, the cultures were first diluted to an $\mathrm{OD}_{600}$ of 0.6 , after

162 which $200 \mu \mathrm{L}$ of the cultures were put into $20 \mathrm{ml}$ of LB solution that included $500 \mathrm{mM} \mathrm{NaCl}, 500$

$163 \mathrm{mM} \mathrm{KCl}$ or $600 \mathrm{mM}$ mannitol and incubated at $37^{\circ} \mathrm{C}$ on a rotary shaker $(150 \mathrm{rpm})$. Then, the

164 bacterial suspension was collected every $2 \mathrm{~h}$ for $24 \mathrm{~h}$, after which the $\mathrm{OD}_{600}$ of the culture was

165 measured. Each experiment was repeated at least 3 times.

\section{Generation of transgenic plants}

167 The pROKII-MdBBX1 recombinant plasmids were transformed into Arabidopsis in accordance

168 with the floral-dip method via Agrobacterium tumefaciens (GV3101)-mediated transformation.

169 Subsequently, the $M d B B X 1$ overexpression seedlings were screened on MS agar media

170 supplemented with $50 \mu \mathrm{g} / \mathrm{mL}$ kanamycin and were further identified via PCR using $M d B B X 1$ and

171 GFP primers. The specific primers used are shown in the Table S1.

172 Analysis of germination status under different abiotic stresses

173 Fifty seeds of WT or overexpression (OE) lines were sown onto 1/2-strength MS agar media

174 supplemented with different concentrations of mannitol (300 or $400 \mathrm{mM}), \mathrm{NaCl}(150$ or $200 \mathrm{mM})$

175 or ABA $(0.2$ or $0.6 \mu \mathrm{M})$. Seed germination was observed and measured every $12 \mathrm{~h}$. For root length

176 analysis, the seeds were grown vertically on 1/2-strength MS media as described above. The root

177 length of 20 seedlings was measured after 10 days, and each treatment was performed at least three

178 times. 
179 Measurements of proline (PRO), malondialdehyde (MDA), and reactive oxygen species (ROS) contents and antioxidant enzyme activity

181 For physiological index measurements, the free PRO content was measured using a

182 spectrophotometric PRO kit (Solarbio Life Sciences, China). The MDA content was measured

183 using a thiobarbituric acid reactive substances assay (Aguilar Díaz de León \& Borges, 2020;

184 Hodges et al., 1999) and the contents of hydrogen peroxide $\left(\mathrm{H}_{2} \mathrm{O}_{2}\right)$ and superoxide anions $\left(\mathrm{O}_{2}{ }^{--}\right)$

185 were measured using $\mathrm{O}_{2}-$ and $\mathrm{H}_{2} \mathrm{O}_{2}$ kits, respectively (Nanjing Jiancheng Bioengineering Institute,

186 China). Similarly, the total protein contents were determined using a BCA Protein Assay Kit

187 (Nanjing Jiancheng Bioengineering Institute, China), and the activities of superoxide dismutase

188 (SOD), peroxidase (POD), catalase (CAT) and ascorbate peroxidase (APX) were measured based

189 on the protocols of the corresponding kits (Nanjing Jiancheng Bioengineering Institute, China)

190 (Bai et al., 2020; Li et al., 2019; Ma et al., 2019).

191 Statistical analysis

192 All experiments were conducted at least three times. The data presented are the means \pm standard

193 deviations of three replications. Statistical significance was analyzed using SPSS software (version

194 17.0), and Turkey's multiple range comparison tests were performed to determine the significance

195 of differences between samples $(\mathrm{P}<0.05$ or $\mathrm{P}<0.01)$.

196 Results

197 Organ-specific expression pattern analysis and subcellular localization of MdBBX1 
198 The organ-specific expression pattern of $M d B B X 1$ was analyzed via qRT-PCR. The results

199 revealed that the transcript level of $M d B B X 1$ was obviously higher in the leaves than in other

200 organs (Fig. 1A). To identify the subcellular localization of MdBBX1, an MdBBX1-GFP construct

201 and empty GFP plasmid were introduced into epidermal cells of tobacco leaves, after which the

202 nuclei were stained by DAPI. The fluorescence signal and DAPI staining were predominantly

203 distributed in the nucleus (Fig. 1B), which indicated that MdBBX1 was localized there.

204 The MdBBX1 promoter contains elements related to the abiotic stress response

205 Some $B B X$ members were found to respond positively to abiotic stresses in a previous study ( $A n$

206 et al., 2020; Crocco \& Botto, 2013; Liu et al., 2018; Shalmani et al., 2018). To explore the potential

207 functions of $M d B B X 1$ in response to a variety of abiotic stresses, the DNA sequence within 2000

208 bp upstream of $M d B B X 1$ (the promoter sequence) was scanned via PlantCARE software

209 (http://bioinformatics.psb.ugent.be-/webtools/plantcare/html/). The results showed that many cis-

210 acting elements that may be involved in responses to abiotic stress, light and other signals were

211 present in the $M d B B X 1$ promoter region (Table 1). For instance, MBS (MYB-binding site)

212 elements are involved in the response to drought stress, and ABA-responsive elements (ABREs)

213 function in response to exogenous ABA. LTRs are found to participate in low-temperature

214 responsiveness. In addition, some cis-acting elements such as TC-rich repeats and TCA elements

215 are involved in responses to defense and stress or to salicylic acid. Taken together, these results

216 indicated that $M d B B X 1$ may participate in the response to abiotic stress. 
217 To further investigate whether $M d B B X 1$ is expressed in response to abiotic stress, apple

218 seedlings were treated with solutions of $100 \mu \mathrm{M}$ ABA, 25\% polyethylene glycol (PEG), or 250

$219 \mathrm{mM} \mathrm{NaCl}$ or $4^{\circ} \mathrm{C}$ for different durations. As shown in Fig. 2, the transcript levels of $M d B B X 1$ were

220 upregulated in both the leaves and the roots under exogenous ABA, salt and PEG treatment.,

$221 M d B B X 1$ expression was maximized after $6 \mathrm{~h}$ of stimulation by ABA, PEG or $\mathrm{NaCl}$ compared

222 with the control in the roots and increased by approximately 120-, 4- and 2-fold, respectively. In

223 addition, the transcript levels of $M d B B X 1$ were upregulated by $12-, 20$ - and 10-fold after treatment

224 with $\mathrm{ABA}, \mathrm{PEG}$ or $\mathrm{NaCl}$ in the leaves. Notably, the expression of $M d B B X 1$ was significantly

225 upregulated under cold conditions only in the roots. Taken together, the above results showed that

226 the expression of $M d B B X 1$ was induced in response to different abiotic stresses, which suggested

227 that $M d B B X 1$ may be involved in the response to abiotic stress.

228 Ectopic expression of MdBBX1 in Escherichia improved cell tolerance to abiotic

229 stress

230 To determine the stress resistance function of $M d B B X 1$, heterogeneous expression of $M d B B X 1$

231 was induced in Escherichia coli growing on solid media under different stress conditions. Survival

232 tests of Escherichia coli cells were carried out, with empty vectors used as controls. As shown in

233 Figure 3A-B, the growth of the cells in nonstress media showed slight significant difference

234 between those harboring $M d B B X 1$ and those harboring the empty vector. However, when the same

235 concentration of cultures was inoculated onto plates with stress media, the number of Escherichia

236 coli colonies expressing $M d B B X 1$ was significantly higher than that of the control colonies 
237 harboring the empty vector. To further confirm the function of $M d B B X 1$, a growth curve of

238 Escherichia coli in liquid media was constructed. As shown in Fig. 3C, under nonstress conditions,

239 few differences were observed among the growth curves of cells with and without $M d B B X 1$

240 expression. However, under different stress conditions, the growth rate of Escherichia coli

241 expressing $M d B B X 1$ was significantly faster than that of the control cells carrying the empty

242 vector. The results suggested that $M d B B X 1$ provided strong abiotic stress tolerance.

243 Overexpression of MdBBX1 increased resistance to abiotic stress in Arabidopsis

244 To determine the role of $M d B B X 1$ in abiotic stress resistance in plants, three transgenic lines (OE1,

245 OE2 and OE3) with similar expression levels of $M d B B X 1$ were obtained and subjected to salt and

246 drought treatment (Fig. S1). As shown in Fig. 4A, on normal media, the germination rate and

247 growth status of seedlings exhibited no obvious differences between the WT and transgenic lines.

248 However, under salt stress, the OE seeds presented a significantly higher germination rate than did

249 the WT seeds. On the stress media that included $200 \mathrm{mM} \mathrm{NaCl}$, the germination rate of the OE

250 seeds reached $60 \%$ compared with $20 \%$ for WT seeds after treatment for $48 \mathrm{~h}$. In addition, the root

251 length of OE lines was obviously longer than that of the WT plants on $150 \mathrm{mM} \mathrm{NaCl}$ media (Fig.

252 4B). When the 3-week-old seedlings were watered with $250 \mathrm{mM} \mathrm{NaCl}$ for 10 days, the leaves of

253 WT began to turn yellow, but few yellow leaves were observed on the OE seedlings. After

254 treatment for 15 days, more wilted and chlorotic leaves were observed on the WT than in the OE

255 lines. After treatment with salt for 20 days, the OE plants presented a significantly higher survival

256 rate (approximately 90\%) than did the WT plants (approximately 40\%) (Fig. 5A). In addition, 
257 under salt stress, the PRO accumulation in the OE plants was obviously higher than that in the WT

258 (Fig. 5B), while the levels of MDA were obviously lower in the OE lines than in the WT (Fig. 5C).

259 Similarly, after mannitol treatment, the OE lines also displayed significantly higher

260 germination rates and longer root lengths than did the WT line (Fig. 6A-B). Moreover, the

261 transgenic plants presented a higher survival rate than did the WT plants when treated with $25 \%$

262 PEG-6000 (Fig. 6C). When treated for 25 days, nearly 60\% of WT plants wilted and died, while

263 the leaves of OE plants yellowed slightly. The survival rate of the OE plants was approximately

$26490 \%$, which was significantly higher than the survival rate of the WT plants. Taken together, these

265 results indicated that overexpression of $M d B B X 1$ enhanced the abiotic stress resistance of the

266 transgenic plants during germination and vegetative stage.

\section{Overexpression of $M d B B X 1$ in Arabidopsis decreased sensitivity to ABA}

268 ABA plays a critical role in the physiological regulation of plant development in seed germination

269 and in abiotic stress responses (Vishwakarma et al., 2017). To determine the potential function of

$270 M d B B X 1$ in response to ABA, seeds of the OE and WT lines were plated on MS media either

271 without or with ABA. Under MS media without ABA, the WT and OE lines displayed similar

272 germination rates. However, under ABA treatment, the germination rates of the OE lines were

273 significantly higher than those of the WT (Fig. 7A). Moreover, after the seedlings grew on MS

274 media with ABA for 10 days, the primary root length of the OE seedlings was obviously greater

275 than that of the WT seedlings (Fig. 7B). To determine the effect on root growth, the seeds of WT

276 and OE were sown on MS media for 2 days first and then transferred to plates with media that 
277 included $0.6 \mu \mathrm{M}$ ABA. The root length of the OE seedlings was still longer than that of the WT

278 seedlings (Fig. S2). The above results suggested that overexpression of $M d B B X 1$ decreased ABA

279 sensitivity in Arabidopsis during the germination and seedling stages.

280 In the ABA signaling pathway, HY5 and ABI5 are crucial for seed germination and seedling

281 development (Chen \& Xiong, 2008; Finkelstein et al., 2002; Finkelstein \& Lynch, 2000b). To

282 determine whether the development of $M d B B X 1$-overexpressing seedlings under abiotic stresses

283 was related to HY5 or ABI5, the transcript levels of $A B I 5$ and $H Y 5$ were measured after ABA

284 treatment. The results revealed that the expression of $A B I 5$ was markedly reduced in the OE plants

285 compared with the WT plants, while HY5 changed only slightly (Fig. 7C). Another OE line

286 overexpressing a different $M d B B X$ family member ( $M d B B X 10)$, which is ABA sensitive, was also

287 evaluated under the same treatment (Liu et al., 2018). As shown in Fig. 7C, the changes in $A B I 5$

288 expression levels were opposite between $M d B B X 1$ and $M d B B X 10$, which was reasonably expected

289 in terms of a response to ABA treatment.

290 Overexpression of MdBBX1 reduced ROS accumulation in transgenic plants

291 Various abiotic stresses often lead to the accumulation of excessive amounts of ROS, particularly

$292 \mathrm{H}_{2} \mathrm{O}_{2}$ and $\mathrm{O}_{2} \cdot{ }^{-}$, which has an important impact on plant growth and development (Mittler et al.,

293 2004). To analyze whether $M d B B X 1$ responds to abiotic stress through the regulation of ROS

294 levels, the accumulation of $\mathrm{O}_{2}{ }^{-}$in WT and OE plants was assessed via nitro blue tetrazolium

295 (NBT) staining. No obvious difference was found between the WT and OE lines. However, under

$296 \mathrm{NaCl}$ and PEG conditions, the OE lines accumulated lower levels of $\mathrm{O}_{2}{ }^{-}$than the WT did (Fig. 
297 8A). Furthermore, the contents of $\mathrm{H}_{2} \mathrm{O}_{2}$ and $\mathrm{O}_{2}{ }^{-}-$were measured, and the results showed that their

298 contents in the OE lines were significantly lower than those in the WT (Fig. 8B, C). Similarly,

299 under normal conditions, the activities of SOD, POD and APX were not notably different between

300 the OE and WT lines. However, after salt treatment, the activities of SOD, POD and APX in the

301 OE lines significantly increased compared with those in the WT lines (Fig. 9). Together, these

302 results suggested that overexpression of $M d B B X 1$ could decrease the accumulation of ROS in

303 transgenic plants by mediating the activities of ROS-scavenging enzymes.

\section{Discussion}

305 Many BBXs are involved in the response to abiotic stresses in plants. For example, AtBBX5 and

$306 A t B B X 24$ are positive regulators that modulate the drought and salt stress resistance in Arabidopsis

307 (Min et al., 2015; Nagaoka \& Takano, 2003). Overexpression of OsBBX25 in Arabidopsis

308 increased the tolerance to abiotic stresses (Liu et al., 2012). Similarly, heterologous, constitutive

309 expression of $C m B B X 22$ in Arabidopsis reduced seed germination and seedling growth under

310 exogenous ABA, but improved plant drought tolerance (Liu et al., 2019b). A recent study found

311 that overexpression of $M d B B X 10$ in Arabidopsis can promote the salt and drought tolerance, and

312 the transgenic seedlings were shown to hypersensitive to exogenous ABA (Liu et al., 2019). In

313 this investigation, overexpression of $M d B B X 1$ also enhanced tolerance to abiotic stresses.

314 However, the phenotype of $M d B B X 1$ overexpression plants was different from that of $M d B B X 10$

315 transgenic plants under exogenous ABA treatment. $M d B B X 10$ overexpressing plants were

316 hypersensitive to exogenous ABA, while the $M d B B X 1$ transgenic plants were insensitive to ABA. 
317 As a pivotal phytohormone, ABA is extensively involved in the regulation of plant growth

318 and development (Wang et al., 2019), especially in response to various abiotic stresses and seed

319 germination (Finkelstein et al., 2002). During the initial stages of germination, the endogenous

320 ABA content in seeds decreases rapidly and markedly after imbibition (Ali-Rachedi et al., 2004;

321 Gubler et al., 2005; Jacobsen et al., 2002). When exogenous ABA is added, seed germination and

322 seedling growth can be repressed (Finkelstein et al., 2002; Finkelstein \& Lynch, 2000a). ABA-

323 insensitive genes $(A B I s)$, especially $A B I 5$, play a vital role in $\mathrm{ABA}$ signaling and

324 photomorphogenesis. ABI5 is mainly expressed in dry seeds, and is involved in ABA-dependent

325 growth arrest when seed dormancy is broken (Finkelstein et al., 2002; Finkelstein \& Lynch,

326 2000b). The efficiency of the ABA-dependent growth arrest is directly dependent on $A B I 5$ levels

327 (Brocard et al., 2002; Lopez-Molina et al., 2001). ABI5 markedly decreases after germination but

328 can be induced by exogenous ABA (Finkelstein \& Lynch, 2000b; Lopez-Molina et al., 2001).

329 Moreover, the expression of $A B I 5$ can be activated by HY5 through direct binding to its promoter

330 in Arabidopsis (Chen et al., 2008). BBX family members also regulate the expression of $A B I 5$.

331 For example, BBX19 from Arabidopsis suppresses seed germination by inducing expression of

332 ABI5 (Bai et al., 2019). However, in this investigation, the transcript level of $A B I 5$ significantly

333 decreased in the $M d B B X 1$ OE plants compared with the WT plants, whereas it was significantly

334 increased in the $M d B B X 10$ overexpression plants (Fig. 7C). Moreover, the transcript levels of $H Y 5$

335 did not obviously change in the $M d B B X 1$ OE seedlings compared with the WT seedlings, but they

336 were obliviously lower than those in the $M d B B X 10$ OE seedlings. These results are consistent with 
337 the phenotypes during seed germination after ABA treatment. In addition, the results of multiple

338 sequence alignment revealed $17 \%, 36 \%$, and 16\% homology between $\mathrm{HY} 5$ and BBX1, BBX5, and

339 BBX21, respectively (Fig. S3), which suggested that overexpression of $M d B B X 1$ may have little

340 effect on the expression of endogenous genes. Taken together, these results suggested that

$341 M d B B X 1$ may interfere with the expression of $A B I 5$ and $H Y 5$ to promote seed germination and

342 seeding growth in transgenic plants.

343 Abiotic stress can disrupt the normal homeostasis of plants, leading to the production of ROS,

344 mainly comprises of $\mathrm{H}_{2} \mathrm{O}_{2}$ and $\mathrm{O}_{2}{ }^{--}$(Miller et al., 2010). Low concentrations of ROS act as critical

345 signaling molecules that are beneficial to plant growth and development, especially when plants

346 are exposed to extreme environmental conditions (Schippers et al., 2012). However, the

347 accumulation of excessive amounts of ROS leads to very serious oxidative damage to plant cells

348 and represses the normal growth of plants (Mullineaux \& Baker, 2010). To alleviate oxidative

349 damage, plant cells immediately employ a series of response mechanisms to suppress ROS

350 production, such as the activation of ROS-scavenging enzymes (SOD, POD, CAT and APX)

351 (Miller et al., 2010). Abiotic stress often increases the accumulation of ROS, thus causing

352 membrane damage with lipid peroxidation, generating MDA. Our results suggested that the ROS

353 and MDA contents were no significant different between the OE and WT lines under normal

354 conditions. However, under salt treatment, compared with the WT line, the MdBBXI transgenic

355 lines displayed a greater ROS-scavenging ability and antioxidant enzyme activities (Fig. 9), and a 
356 lower ROS accumulation (Fig. 8) and MDA levels (Fig. 5). Regulation of antioxidant capacity

357 through improving the ROS-scavenging system might be a common mechanism to increase salt

358 tolerance. Similar to our study, a previous study reported that overexpression of ThSOS3 from

359 Tamarix hispida improved the salt tolerance of transgenic plants by alleviating the accumulation

360 of ROS, decreasing the accumulation of MDA accumulation and increasing the activity of

361 antioxidant enzymes (Liu et al., 2020). In addition, overexpression of apple MdMIPS1 also

362 enhanced salt tolerance by increasing the activities of SOD, POD, and decreasing ROS and MDA

363 contents in transgenic apple under salt stress (Hu et al., 2020). Taken together, these results

364 indicated that $M d B B X 1$ provides salt stress resistance by enhancing ROS-scavenging system and

365 alleviating oxidative stress.

366 Conclusions

367 The transcript level of $M d B B X 1$ increased in response to various stresses. Overexpressing

$368 M d B B X 1$ in Arabidopsis improved abiotic stress tolerance by regulating ABA signaling and the

369 production of ROS. However, the detailed molecular mechanisms underlying these phenomena

370 still need to be tested in future experiments.

371 Acknowledgements

372 The present study was supported by National Natural Science Foundation of China (grant number

37331872042 and 31972358), the Natural Science Foundation of Shandong Province, China (grant

374 number ZR2019MC040 and ZR2018MC022), and Shandong Provincial Key Research and

375 Development Project (grant number 2019JZZY010727 and 2019 GNC106147).

Peer] reviewing PDF | (2021:09:65406:3:0:NEW 23 Dec 2021) 
376

377

378

379

380

381

382

383

384

385

386

387

388

389

390

391

392

393

394

395

396

397

398

399

400

\section{Reference}

Aguilar Díaz de León J, and Borges CR. 2020. Evaluation of oxidative stress in biological samples using the thiobarbituric acid reactive substances assay. $J$ Vis Exp 159: DOI 10.3791/61122.

Ali-Rachedi S, Bouinot D, Wagner MH, Bonnet M, Sotta B, Grappin P, and Jullien M. 2004. Changes in endogenous abscisic acid levels during dormancy release and maintenance of mature seeds: studies with the Cape Verde Islands ecotype, the dormant model of Arabidopsis thaliana. Planta 219:479-488 DOI 10.1007/s00425-004-1251-4.

An JP, Wang XF, Espley RV, Lin-Wang K, Bi SQ, You CX, and Hao YJ. 2020. An apple BBox protein MdBBX37 modulates anthocyanin biosynthesis and hypocotyl elongation synergistically with MdMYBs and MdHY5. Plant Cell Physiol 61:130-143 DOI $10.1093 / \mathrm{pcp} / \mathrm{pcz} 185$.

Bai M, Sun J, Liu J, Ren H, Wang K, Wang Y, Wang C, and Dehesh K. 2019. The B-box protein BBX19 suppresses seed germination via induction of ABI5. Plant J 99:1192-1202 DOI $10.1111 /$ tpj.14415.

Bai Y, Xiao S, Zhang Z, Zhang Y, Sun H, Zhang K, Wang X, Bai Z, Li C, and Liu L. 2020. Melatonin improves the germination rate of cotton seeds under drought stress by opening pores in the seed coat. PeerJ 8: e9450 DOI 10.7717/peerj.9450.

Brocard IM, Lynch TJ, and Finkelstein RR. 2002. Regulation and role of the Arabidopsis abscisic acid-insensitive 5 gene in abscisic acid, sugar, and stress response. Plant Physiol 129:1533-1543 DOI 10.1104/pp.005793.

Chang CS, Li YH, Chen LT, Chen WC, Hsieh WP, Shin J, Jane WN, Chou SJ, Choi G, Hu JM, Somerville S, and Wu SH. 2008. LZF1, a HY5-regulated transcriptional factor, functions in Arabidopsis de-etiolation. Plant $J$ 54:205-219 DOI 10.1111/j.1365313X.2008.03401.x. 
401 Chen H, and Xiong L. 2008. Role of HY5 in abscisic acid response in seeds and seedlings. Plant $402 \quad$ Signal Behav 3:986-988 DOI 10.4161/psb.6185.

403 Chen H, Zhang J, Neff MM, Hong SW, Zhang H, Deng XW, and Xiong L. 2008. Integration 404 of light and abscisic acid signaling during seed germination and early seedling 405 development. Proc Natl Acad Sci US A 105:4495-4500 DOI 10.1073/pnas.0710778105.

406

407

408

409

410

411

412

413

414

415

416

417

418

419

420

421

422

423

424

425

426

Chen J, Chen JY, Wang JN, Kuang JF, Shan W, and Lu WJ. 2012. Molecular characterization and expression profiles of MaCOL1, a CONSTANS-like gene in banana fruit. Gene 496:110-117 DOI 10.1016/j.gene.2012.01.008.

Chu Z, Wang X, Li Y, Yu H, Li J, Lu Y, Li H, and Ouyang B. 2016. Genomic organization, phylogenetic and expression analysis of the B-BOX gene family in tomato. Front Plant Sci 7:1552 DOI 10.3389/fpls.2016.01552.

Crocco CD, and Botto JF. 2013. BBX proteins in green plants: Insights into their evolution, structure, feature and functional diversification. Gene 531:44-52 DOI 10.1016/j.gene.2013.08.037.

Crocco CD, Holm M, Yanovsky MJ, and Botto JF. 2011. Function of B-BOX under shade. Plant Signal Behav 6:101-104 DOI 10.4161/psb.6.1.14185.

Crocco CD, Locascio A, Escudero CM, Alabadi D, Blazquez MA, and Botto JF. 2015. The transcriptional regulator BBX24 impairs DELLA activity to promote shade avoidance in Arabidopsis thaliana. Nat Commun 6:6202 DOI 10.1038/ncomms7202.

Crocco CD, Ocampo GG, Ploschuk EL, Mantese A, and Botto JF. 2018. Heterologous Expression of AtBBX21 enhances the rate of photosynthesis and alleviates photoinhibition in Solanum tuberosum. Plant Physiol 177:369-380 DOI 10.1104/pp.17.01417.

Datta S, Hettiarachchi C, Johansson H, and Holm M. 2007. SALT TOLERANCE HOMOLOG2, a B-box protein in Arabidopsis that activates transcription and positively regulates light-mediated development. Plant Cell 19:3242-3255 DOI 10.1105/tpc.107.054791. 
427

428

429

430

431

432

433

434

435

436

437

438

439

440

441

442

443

444

445

446

447

448

449

450

451

Datta S, Johansson H, Hettiarachchi C, Irigoyen ML, Desai M, Rubio V, and Holm M. 2008. LZF1/SALT TOLERANCE HOMOLOG3, an Arabidopsis B-box protein involved in light-dependent development and gene expression, undergoes COP1-mediated ubiquitination. Plant Cell 20:2324-2338 DOI 10.1105/tpc.108.061747.

Du F, Xu JN, Zhan CY, Yu ZB, and Wang XY. 2014. An obesity-like gene MdTLP7 from apple (Malus x domestica) enhances abiotic stress tolerance. Biochem Biophys Res Commun 445:394-397 DOI 10.1016/j.bbrc.2014.02.005.

Fang H, Dong Y, Yue X, Hu J, Jiang S, Xu H, Wang Y, Su M, Zhang J, Zhang Z, Wang N, and Chen X. 2019. The B-box zinc finger protein MdBBX20 integrates anthocyanin accumulation in response to ultraviolet radiation and low temperature. Plant Cell Environ 42:2090-2104 DOI 10.1111/pce.13552.

Finkelstein RR, Gampala SS, and Rock CD. 2002. Abscisic acid signaling in seeds and seedlings. Plant Cell 14 Suppl:S15-45 DOI 10.1105/tpc.010441.

Finkelstein RR, and Lynch TJ. 2000a. Abscisic acid inhibition of radicle emergence but not seedling growth is suppressed by sugars. Plant Physiol 122:1179-1186 DOI 10.1104/pp.122.4.1179.

Finkelstein RR, and Lynch TJ. 2000b. The Arabidopsis abscisic acid response gene ABI5 encodes a basic leucine zipper transcription factor. Plant Cell 12:599-609 DOI $10.2307 / 3871072$.

Gangappa SN, and Botto JF. 2014. The BBX family of plant transcription factors. Trends Plant Sci 19:460-470 DOI 10.1016/j.tplants.2014.01.010.

Gangappa SN, Crocco CD, Johansson H, Datta S, Hettiarachchi C, Holm M, and Botto JF. 2013. The Arabidopsis B-BOX protein BBX25 interacts with HY5, negatively regulating BBX22 expression to suppress seedling photomorphogenesis. Plant Cell 25:1243-1257 DOI 10.1105/tpc.113.109751. 
452 Gasic K, Hernandez A, and Korban SSJPMBR. 2004. RNA extraction from different apple

453

454

455

456

457

458

459

460

461

462

463

464

465

466

467

468

469

470

471

472

473

474

475

476 tissues rich in polyphenols and polysaccharides for cDNA library construction. Plant Molecular Biology Reporter 22:437-438 DOI 10.1007/BF02772687.

Gubler F, Millar AA, and Jacobsen JV. 2005. Dormancy release, ABA and pre-harvest sprouting. Curr Opin Plant Biol 8:183-187 DOI 10.1016/j.pbi.2005.01.011.

Heng Y, Jiang Y, Zhao X, Zhou H, Wang X, Deng XW, and Xu D. 2019a. BBX4, a phyBinteracting and modulated regulator, directly interacts with PIF3 to fine tune red lightmediated photomorphogenesis. Proc Natl Acad Sci USA 116:26049-26056 DOI 10.1073/pnas.1915149116.

Heng Y, Lin F, Jiang Y, Ding M, Yan T, Lan H, Zhou H, Zhao X, Xu D, and Deng XW. 2019b. B-Box Containing Proteins BBX30 and BBX31, Acting Downstream of HY5, Negatively Regulate Photomorphogenesis in Arabidopsis. Plant Physiol 180:497-508 DOI $10.1104 /$ pp.18.01244.

Hodges DM, Delong JM, and Prange F. 1999. Improving the thiobarbituric acid-reactivesubstances assay for estimating lipid peroxidation in plant tissues containing anthocyanin and other interfering compounds. Planta 207:604-611 DOI 10.1007/s004250050524.

Hu L, Zhou K, Liu Y, Yang S, Zhang J, Gong X, and Ma F. 2020. Overexpression of MdMIPS1 enhances salt tolerance by improving osmosis, ion balance, and antioxidant activity in transgenic apple. Plant Science 301:110654 DOI 10.1016/j.plantsci.2020.110654.

Jacobsen JV, Pearce DW, Poole AT, Pharis RP, and Mander LN. 2002. Abscisic acid, phaseic acid and gibberellin contents associated with dormancy and germination in barley. Physiol Plant 115:428-441 DOI 10.1034/j.1399-3054.2002.1150313.x.

Job N, Yadukrishnan P, Bursch K, Datta S, and Johansson H. 2018. Two B-Box proteins regulate photomorphogenesis by oppositely modulating HY5 through their diverse Cterminal domains. Plant Physiol 176:2963-2976 DOI 10.1104/pp.17.00856. 
Khanna R, Kronmiller B, Maszle DR, Coupland G, Holm M, Mizuno T, and Wu SH. 2009. The Arabidopsis B-box zinc finger family. Plant Cell 21:3416-3420 DOI 10.1105/tpc.109.069088.

Li PT, Rashid MHO, Chen TT, Lu QW, Ge Q, Gong WK, Liu AY, Gong JW, Shang HH, Deng XY, Li JW, Li SQ, Xiao XH, Liu RX, Zhang Q, Duan L, Zou XY, Zhang Z, Jiang X, Zhang Y, Peng RH, Shi YZ, and Yuan YL. 2019. Transcriptomic and biochemical analysis of upland cotton (Gossypium hirsutum) and a chromosome segment substitution line from G. hirsutum x G. barbadense in response to Verticillium dahliae infection. BMC Plant Biol 19:19 DOI 10.1186/s12870-018-1619-4.

Liu X, Li R, Dai Y, Chen X, and Wang X. 2018. Genome-wide identification and expression analysis of the B-box gene family in the Apple (Malus domestica Borkh.) genome. Mol Genet Genomics 293:303-315 DOI 10.1007/s00438-017-1386-1.

Liu X, Li R, Dai Y, Yuan L, Sun Q, Zhang S, and Wang X. 2019. A B-box zinc finger protein, MdBBX10, enhanced salt and drought stresses tolerance in Arabidopsis. Plant Molecular Biology 99:437-447 DOI 10.1007/s11103-019-00828-8.

Liu Y, Xing L, Li J, and Dai S. 2012. Rice B-box zinc finger protein OsBBX25 is involved in the abiotic response. Zhiwu Xuebao 47:366-378 DOI 10.3724/SP.J.1259.2012.00366.

Liu Z, Xie Q, Tang F, Wu J, Dong W, Wang C, and Gao C. 2020. The ThSOS3 Gene Improves the salt tolerance of transgenic Tamarix hispida and Arabidopsis thaliana. Frontiers of Plant Science 11:597480 DOI 10.3389/fpls.2020.597480.

Livak KJ, and Schmittgen TD. 2001. Analysis of relative gene expression data using real-time quantitative PCR and the 2(-Delta Delta C(T)) Method. Methods 25:402-408 DOI 10.1006/meth.2001.1262.

Lopez-Molina L, Mongrand S, and Chua NH. 2001. A postgermination developmental arrest checkpoint is mediated by abscisic acid and requires the ABI5 transcription factor in Arabidopsis. Proc Natl Acad Sci U S A 98:4782-4787 DOI 10.1073/pnas.081594298. 
503 Ma Y, Wang P, Wang M, Sun M, Gu Z, and Yang R. 2019. GABA mediates phenolic 504 compounds accumulation and the antioxidant system enhancement in germinated hulless 505 506

Miller G, Suzuki N, Ciftci-Yilmaz S, and Mittler R. 2010. Reactive oxygen species homeostasis 507 and signalling during drought and salinity stresses. Plant Cell Environ 33:453-467 DOI 10.1111/j.1365-3040.2009.02041.x.

Min JH, Chung JS, Lee KH, and Kim CS. 2015. The CONSTANS-like 4 transcription factor, AtCOL4, positively regulates abiotic stress tolerance through an abscisic acid-dependent manner in Arabidopsis. J Integr Plant Biol 57:313-324 DOI 10.1111/jipb.12246.

Mittler R, Vanderauwera S, Gollery M, and Van Breusegem F. 2004. Reactive oxygen gene network of plants. Trends Plant Sci 9:490-498 DOI 10.1016/j.tplants.2004.08.009.

Mullineaux PM, and Baker NR. 2010. Oxidative stress: antagonistic signaling for acclimation or cell death? Plant Physiology 154:521-525 DOI 10.1104/pp.110.161406.

Nagaoka S, and Takano T. 2003. Salt tolerance-related protein STO binds to a $M y b$ transcription factor homologue and confers salt tolerance in Arabidopsis. J Exp Bot 54:2231-2237 DOI

Sarmiento F. 2013. The BBX subfamily IV: additional cogs and sprockets to fine-tune lightdependent development. Plant Signal Behav 8:e23831 DOI 10.4161/psb.23831.

Schippers JH, Nguyen HM, Lu D, Schmidt R, and Mueller-Roeber B. 2012. ROS homeostasis KM, and Han M. 2018. Genome identification of $B-B O X$ gene family members in seven during development: an evolutionary conserved strategy. Cellular and Molecular Life Sciences 69:3245-3257 DOI 10.1007/s00018-012-1092-4. Rosaceae species and their expression qnalysis in response to flower induction in Malus domestica. Molecules 23(7):1763. DOI 10.3390/molecules23071763. 
528

529

530

531

532

533

534

535

536

537

538

539

540

541

542

543

544

545

546

547

548

549

550

551

552

Vishwakarma K, Upadhyay N, Kumar N, Yadav G, Singh J, Mishra RK, Kumar V, Verma R, Upadhyay RG, Pandey M, and Sharma S. 2017. Abscisic acid signaling and abiotic stress tolerance in plants: A review on current knowledge and future prospects. Front Plant Sci 8:161 DOI 10.3389/fpls.2017.00161.

Wang C, He X, Li Y, Wang L, Guo X, and Guo X. 2018. The cotton MAPK kinase GhMPK20 negatively regulates resistance to Fusarium oxysporum by mediating the MKK4-MPK20WRKY40 cascade. Molecular Plant Pathology 19:1624-1638 DOI 10.1111/mpp.12635.

Wang H, Zhang Z, Li H, Zhao X, Liu X, Ortiz M, Lin C, and Liu B. 2013. CONSTANS-LIKE 7 regulates branching and shade avoidance response in Arabidopsis. J Exp Bot 64:10171024 DOI 10.1093/jxb/ers376.

Wang Y, Yang L, Chen X, Ye T, Zhong B, Liu R, Wu Y, and Chan Z. 2016. Major latex protein-like protein 43 (MLP43) functions as a positive regulator during abscisic acid responses and confers drought tolerance in Arabidopsis thaliana. Journal of Experimental Botany 67:421-434 DOI 10.1093/jxb/erv477.

Wang YY, Xiong F, Ren QP, and Wang XL. 2019. Regulation of flowering transition by alternative splicing: the role of the U2 auxiliary factor. $J$ Exp Bot 71(3):751-758. DOI 10.1093/jxb/erz416.

Wei CQ, Chien CW, Ai LF, Zhao J, Zhang Z, Li KH, Burlingame AL, Sun Y, and Wang ZY. 2016. The Arabidopsis B-box protein BZS1/BBX20 interacts with HY5 and mediates strigolactone regulation of photomorphogenesis. J Genet Genomics 43:555-563 DOI 10.1016/j.jgg.2016.05.007.

Yadav A, Bakshi S, Yadukrishnan P, Lingwan M, Dolde U, Wenkel S, Masakapalli SK, and Datta S. 2019. The B-Box-Containing microprotein miP1a/BBX31 regulates photomorphogenesis and UV-B protection. Plant Physiol 179:1876-1892 DOI $10.1104 /$ pp. 18.01258 . 
553 Yuan H, Zhao K, Lei H, Shen X, Liu Y, Liao X, and Li T. 2013. Genome-wide analysis of the 554 GH3 family in apple (Malus x domestica). BMC Genomics 14:297 DOI 10.1186/1471555 2164-14-297.

556 Zhang X, Huai J, Shang F, Xu G, Tang W, Jing Y, and Lin R. 2017. A PIF1/PIF3-HY5-BBX23 DOI 10.1104/pp.17.00418.

559

560

561

562

563

564

565

566

567

568

569

570

571

572

573

574

575

576

577

578

579

580

581

582

583

584

585

586

587

588 
589 Table

590 Table 1 Putative $c i s$-acting elements of the promoter of $M d B B X 1$

591

592 Figure Legends

593 Figure 1 Expressional pattern and subcellular localization analysis of MdBBX1. (A) qRT-

594 PCR analysis of $M d B B X 1$ expression in different organs of six-year old apple seedlings. The

595 experiments were repeated three times and vertical bars indicate the standard error of the mean.

596 The letters above the columns represent significant differences $(\mathrm{P}<0.05)$ based on Tukey's

597 multiple test. (B) Subcellular localization of MdBBX1-GFP fusion protein. 35S::MdBBX1-GFP

598 construct was transformed into tobacco leaves and was examined in the epidermal cells at $48 \mathrm{~h}$

599 after the transformation by confocal fluorescence microscopy. The nuclei were stained with 100

$600 \mathrm{~g} / \mathrm{ml}$ DAPI for $10 \mathrm{~min}$.

601 Figure 2 The expression pattern of $M d B B X 1$ in roots (A) and leaves (B) under various abiotic

602 stresses. Each column represents the mean values of three biological replicates and vertical bars

603 indicate the standard error of the mean. The letters above the columns represent significant

604 differences $(\mathrm{P}<0.05)$ based on Tukey's multiple test.

605 Figure 3 Survival test of $E$. coli cells carrying $M d B B X 1$ or empty vector under various stress

606 conditions. (A) $10 \mu \mathrm{L}$ cultures induced by $1 \mathrm{mM}$ IPTG for $2 \mathrm{~h}$ (OD600 about 0.5 ) were diluted

607 from $10^{-3}$ to $10^{-5}$ and were spotted on solid medium containing $\mathrm{NaCl}, \mathrm{KCl}$ or mannitol. Each

608 experiment was carried out in three biological replicates. (B) The colony numbers appearing on 
609 above medium were counted in $10^{-5}$ concentration. Mean values are from three independent

610 replicates and error bars indicate standard deviation. The letters above the columns represent

611 significant differences $(\mathrm{P}<0.05)$ based on Tukey's multiple test. $(\mathrm{C})$ Growth curve of $E$. coli cells

612 in LB liquid medium with and without addition of $1 \mathrm{mM}$ IPTG under $\mathrm{NaCl}, \mathrm{KCl}$ or mannitol

613 treatments. The mean expression value was calculated from three independent replicates. Vertical

614 bars indicate the standard error of mean, ** and * indicate significant differences compared with

615 vector cells at $\mathrm{P}<0.01$ and $\mathrm{P}<0.05$, respectively.

616 Figure 4 Germination and root length phenotypes of $M d B B X 1$ overexpression plants under

617 salt tolerance. (A) Germination phenotype of the wild type (WT) and $M d B B X 1$-overexpressed

618 (OE) lines under 1/2 MS medium containing $\mathrm{NaCl}(0,150$ and $200 \mathrm{mM})$. Three independent

619 experiments were conducted and each phenotype included 50 seeds. The mean expression value

620 was calculated from three independent replicates. Vertical bars indicate the standard error of mean,

$621 * * P<0.01$ and $* P<0.05$ compared with WT. (B) The root length of WT and $M d B B X 1$-transgenic

622 lines in $1 / 2 \mathrm{MS}$ medium containing $150 \mathrm{mM} \mathrm{NaCl}$. Root growth was measured after $\mathrm{NaCl}$

623 treatment for14 days. The letters above the columns represents significant differences $(\mathrm{P}<0.05)$

624 based on Tukey's multiple test.

625 Figure 5 Overexpression $\boldsymbol{M d B B} \boldsymbol{X} 1$ enhanced salt tolerance in transgenic plants. (A)The

626 representative phenotypes of 3-week old WT and OE seedlings were treated with $\mathrm{NaCl}(250 \mathrm{mM})$

627 for 3 days to 20 days. (B) Survival rates of WT and transgenic plants after salt stress. PRO (C) and

628 MDA content (D) were measured in WT and transgenic plants after salt stress. Mean values are 
629 from three independent replicates and error bars indicate standard deviation. The letters above the

630 columns represent significant differences $(\mathrm{P}<0.05)$ based on Tukey's multiple test.

631 Figure 6 The phenotype of WT and MdBBX1 transgenic plants in response to drought stress.

632 (A) The seed germination of WT and the $M d B B X 1$ transgenic plants in 1/2 MS medium containing

633 mannitol $(0,300$, or $400 \mathrm{mM})$. Three independent experiments were conducted and each

634 phenotype included 50 seeds. Vertical bars indicate the standard error of mean, ** and * indicate

635 significant differences in comparison with $\mathrm{WT}$ at $\mathrm{P}<0.01$ and $\mathrm{P}<0.05$, respectively. (B) The root

636 length of WT and the transgenic plants of $M d B B X 1$ in $1 / 2 \mathrm{MS}$ medium containing $300 \mathrm{mM}$

637 mannitol. Root growth of WT and the transgenic plants of $M d B B X 1$ was measured after 14 days.

638 The letters above the columns represent significant differences $(\mathrm{P}<0.05)$ based on Tukey's

639 multiple test. (C) The representative phenotypes of WT and OE seedlings were treated with $25 \%$

640 (w/v) PEG-6000 for 10 days and 25 days. All the columns were represented as mean values of

641 three independent replicates and error bars indicate standard deviation. The letters above the

642 columns represent significant differences $(\mathrm{P}<0.05)$ based on Tukey's multiple test.

643 Figure 7 Germination phenotype of WT and transgenic plants in response to ABA. (A) Seed

644 germination of WT and OE lines in 1/2 MS medium containing different ABA concentrations $(0$,

6450.2 or $0.6 \mu \mathrm{M})$. Three independent experiments were conducted and each phenotype included 50

646 seeds. Vertical bars indicate the standard error of mean, ${ }^{* *}$ and $*$ indicate significant differences

647 in comparison with WT at $\mathrm{P}<0.01$ and $\mathrm{P}<0.05$, respectively. (B) The root length of WT and $\mathrm{OE}$

648 lines in 1/2 MS medium containing 0.6 $\mu \mathrm{M}$ ABA. Root growth of WT and OE seedlings was 
649 measured after 14 days. (C) The transcriptional levels of HY5 and ABI5 in WT and OE lines after

$650100 \mu \mathrm{M}$ ABA treatment for $3 \mathrm{~h}$. Little difference was observed among different lines, so one of

651 the $\mathrm{OE} M d B B X 1$ transgenic lines was selected as the representative for gene expression analysis.

652 The letters above the columns represent significant differences $(\mathrm{P}<0.05)$ based on Tukey's

653 multiple test.

654 Figure 8 Analysis of ROS in WT and the MdBBX1 transgenic plants after salt and drought

655 stress. (A) NBT staining of $\mathrm{O}_{2}{ }^{--}$in WT and OE plants after the treatment with NaCl or PEG-6000

656 for $6 \mathrm{~h}$. (B) The content of $\mathrm{H}_{2} \mathrm{O}_{2}$ and $\mathrm{O}_{2}{ }^{-}$in WT and transgenic lines after $\mathrm{NaCl}$ treatment. Each

657 column represents the average of three replicates and error bars indicate standard deviation. The

658 letters above the columns represent significant differences $(\mathrm{P}<0.05)$ based on Tukey's multiple 659 test.

660 Figure 9 The activities of ROS-scavenging enzymes in WT and the MdBBX1 transgenic

661 plants after NaCl treatment. Each column represents the average of three replicates and error

662 bars indicate standard deviation. The letters above the columns represent significant differences

$663(\mathrm{P}<0.05)$ based on Tukey's multiple test.

664

665

666

667

668 


\section{Supplementary materials}

670 Table S1 Primers used in this experiment.

671 Figure S1 Identification of the transgenic plants of $\boldsymbol{M d B B X 1}$. A. Phenotype of $\mathrm{OE}_{1}, \mathrm{OE}_{2}$ and

$672 \mathrm{OE}_{3}$ plants; B. PCR products of transgenic plants; C. The expression of $M d B B X 1$ in the leaves of

673 WT and transgenic plants.

674 Figure S2 Roots length of WT and OE seedlings in medium containing ABA. The wild type

675 and OE seedlings were grown on MS for 2 days and then were transferred to $1 / 2$ MS medium

676 containing $0.6 \mu \mathrm{M}$ ABA.

677 Figure S3 The homology among MdBBX1, AtBBX1, AtBBX5 and AtBBX21. Only 17\%, 36\%,

$67816 \%$ homology between MdBBX1 and AtBBX1, AtBBX5, and AtBBX21, respectively. 
Table $\mathbf{1}$ (on next page)

Putative cis-acting elements of the promoter of MdBBXI 
1 Table 1. Putative cis-acting elements of the promoter of $M d \mathrm{BBX} 1$

\begin{tabular}{llll}
\hline Cis-element & Position & Sequence $\left(5^{\prime}-\right.$ & Function \\
& & $\left.3^{\prime}\right)$ & \\
\hline ABRE & -1331 & ACGTG & cis-acting element involved in the abscisic acid responsiveness \\
CGTCA-motif & -1794 & CGTCA & cis-acting regulatory element involved in the MeJA-responsiveness \\
G-Box & -1330 & CACGTT & cis-acting regulatory element involved in light responsiveness \\
GT1-motif & -1523 & GGTTAAT & light responsive element \\
LTR & -1992 & CCGAAA & cis-acting element involved in low-temperature responsiveness \\
MBS & -577 & CAACTG & MYB binding site involved in drought-inducibility \\
P-box & -1778 & CCTTTTG & gibberellin-responsive element \\
TC-rich repeats & -941 & ATTCTCTAAC & cis-acting element involved in defense and stress responsiveness \\
TCA-element & -1242 & CCATCTTTTT & cis-acting element involved in salicylic acid responsiveness \\
\hline
\end{tabular}

2 


\section{Figure 1}

Expressional pattern and subcellular localization analysis of MdBBX1.

(A) qRT-PCR analysis of MdBBX1 expression in different organs of six-year old apple seedlings. The experiments were repeated three times and vertical bars indicate the standard error of the mean. The letters above the columns represent significant differences $(P<0.05)$ based on Tukey's multiple test. (B) Subcellular localization of MdBBX1-GFP fusion protein. 35S::MdBBX1-GFP construct was transformed into tobacco leaves and was examined in the epidermal cells at $48 \mathrm{~h}$ after the transformation by confocal fluorescence microscopy. The nuclei were stained with $100 \mathrm{~g} / \mathrm{ml}$ DAPI for $10 \mathrm{~min}$. 
A

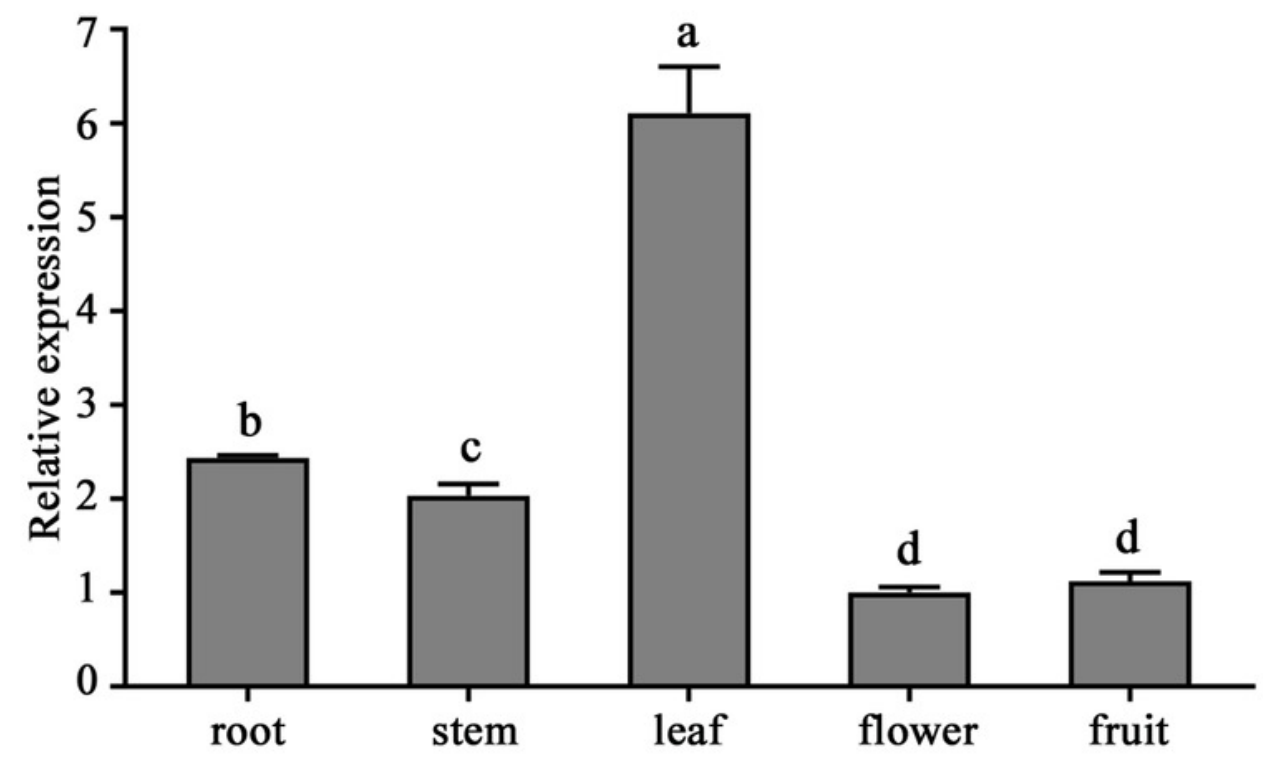

B

Bright
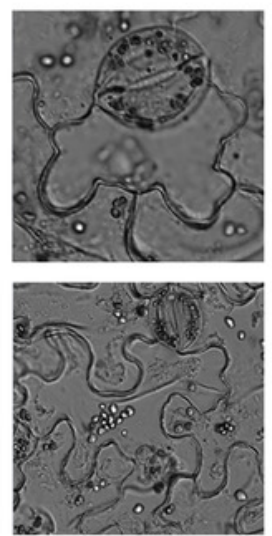

DAPI
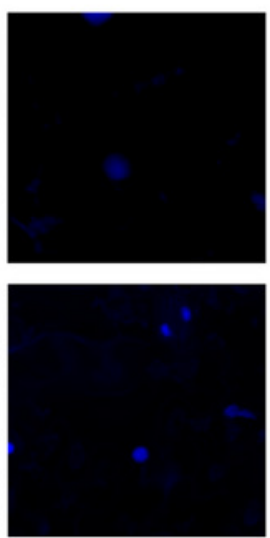

Chloroplast
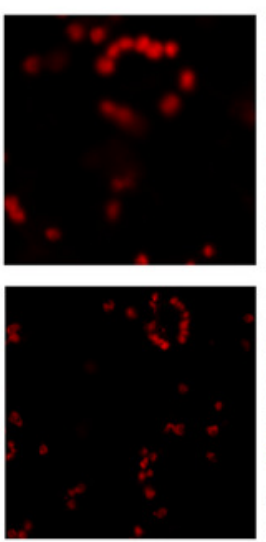

Merge
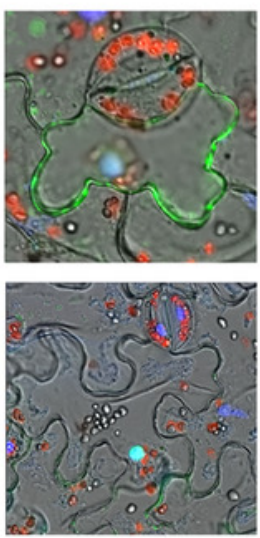
Figure 2

The expression pattern of $M d B B X 1$ in roots and leaves under various abiotic stresses.

Each column represents the mean values of three biological replicates and vertical bars indicate the standard error of the mean. The letters above the columns represent significant differences $(P<0.05)$ based on Tukey's multiple test. 

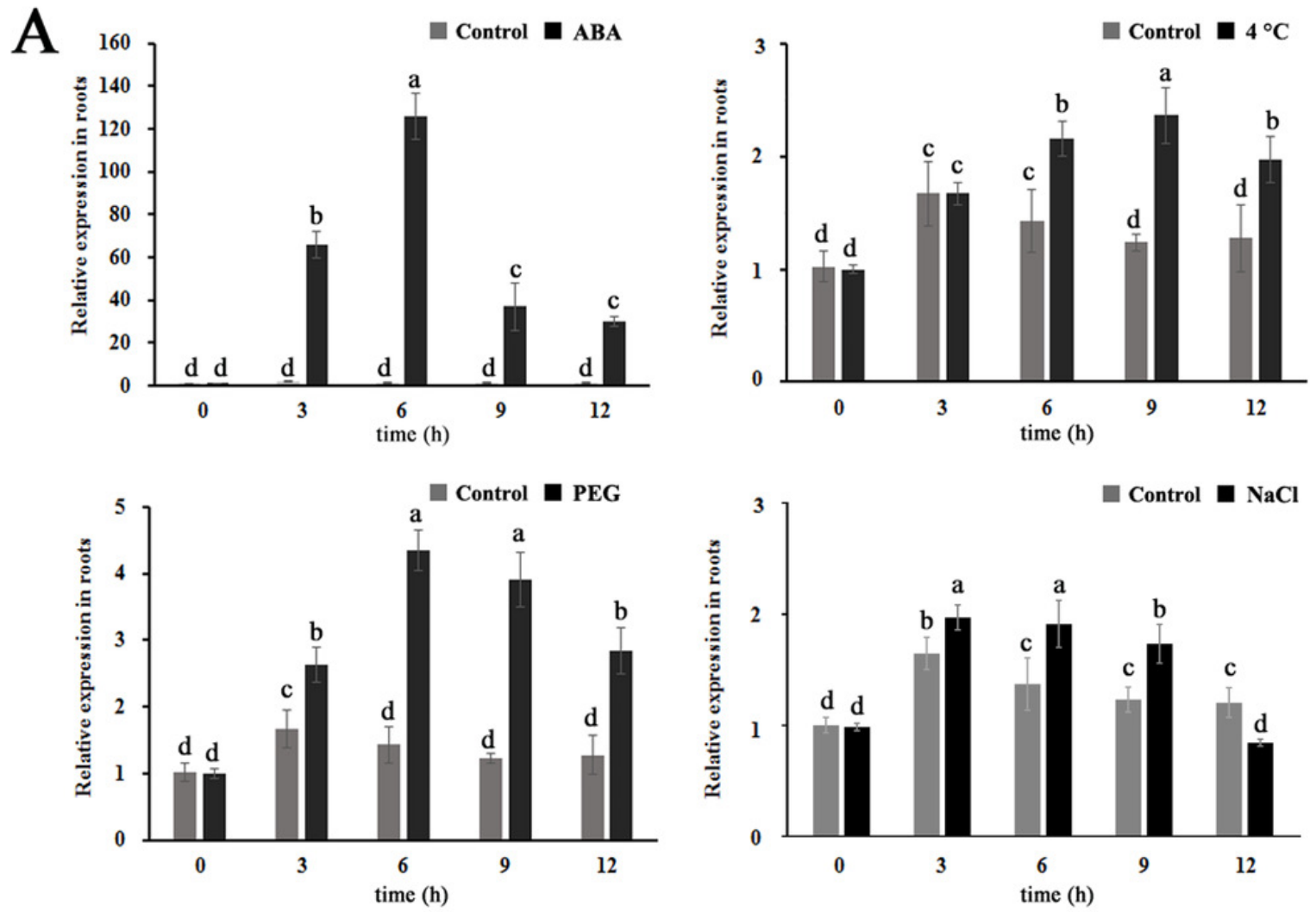

B
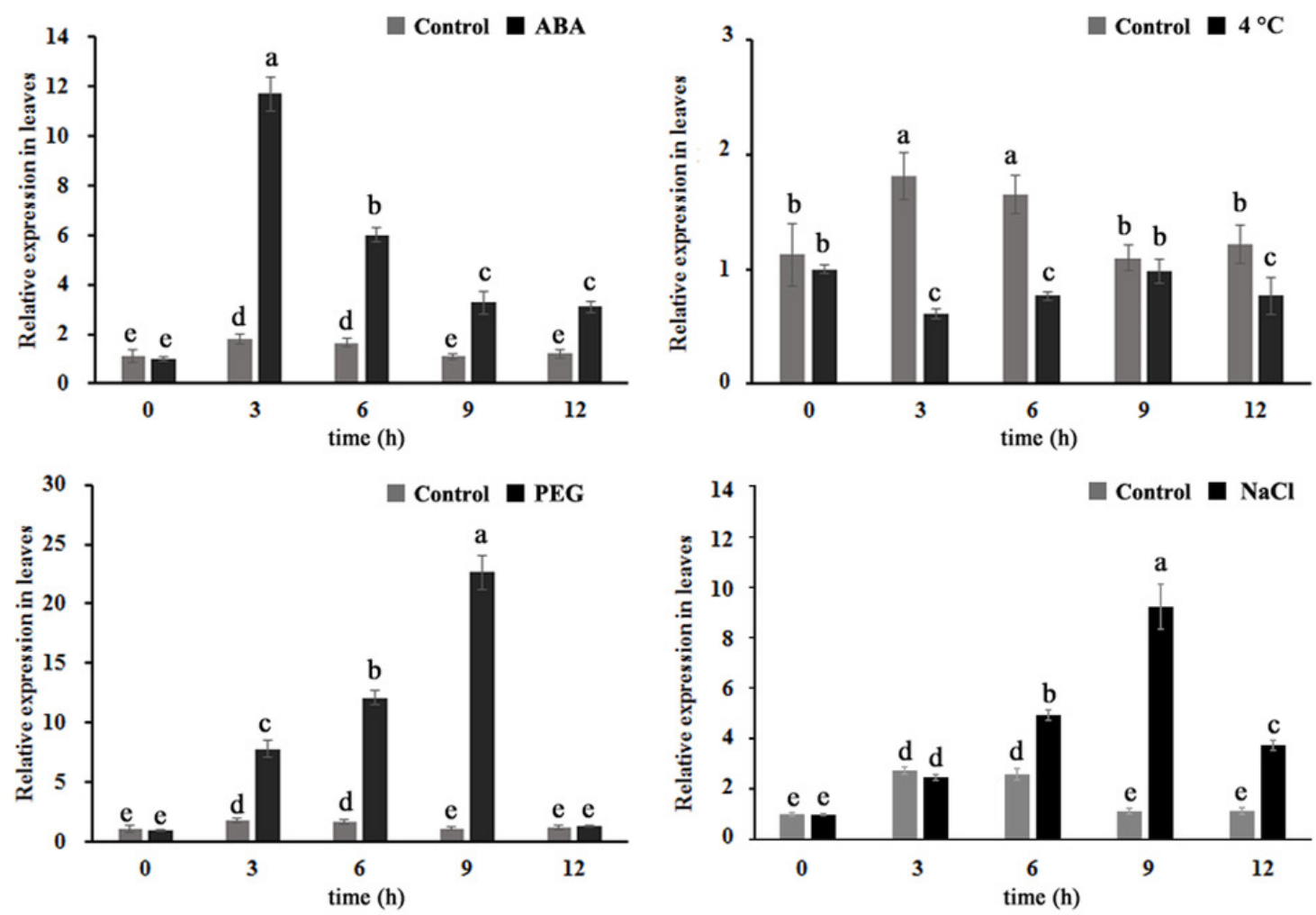


\section{Figure 3}

Survival test of $E$. coli cells carrying MdBBXI or empty vector under various stress conditions.

(A) $10 \mu \mathrm{L}$ cultures induced by $1 \mathrm{mM} \mathrm{IPTG} \mathrm{for} 2 \mathrm{~h}$ (OD600 about 0.5 ) were diluted from $10^{-3}$ to $10^{-5}$ and were spotted on solid medium containing $\mathrm{NaCl}, \mathrm{KCl}$ or mannitol. Each experiment was carried out in three biological replicates. (B) The colony numbers appearing on above medium were counted in $10^{-5}$ concentration. Mean values are from three independent replicates and error bars indicate standard deviation. The letters above the columns represent significant differences ( $P<0.05)$ based on Tukey's multiple test. (C) Growth curve of $E$. coli cells in LB liquid medium with and without addition of $1 \mathrm{mM} \mathrm{IPTG}$ under $\mathrm{NaCl}, \mathrm{KCl}$ or mannitol treatments. The mean expression value was calculated from three independent replicates. Vertical bars indicate the standard error of mean, ${ }^{* *}$ and $*$ indicate significant differences compared with vector cells at $\mathrm{P}<0.01$ and $\mathrm{P}<0.05$, respectively. 
A
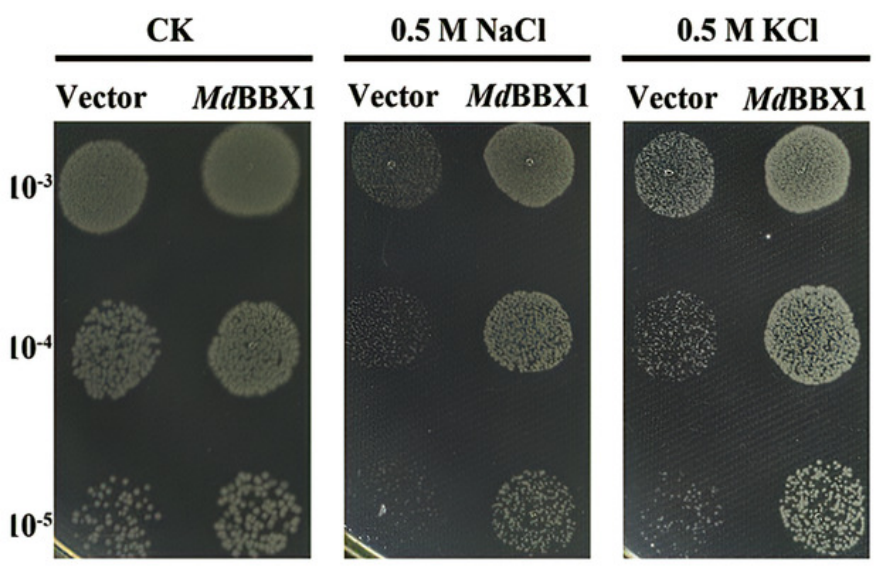

$\frac{0.6 \mathrm{M} \text { Mannitol }}{\text { Vector MdBBX1 }}$

B

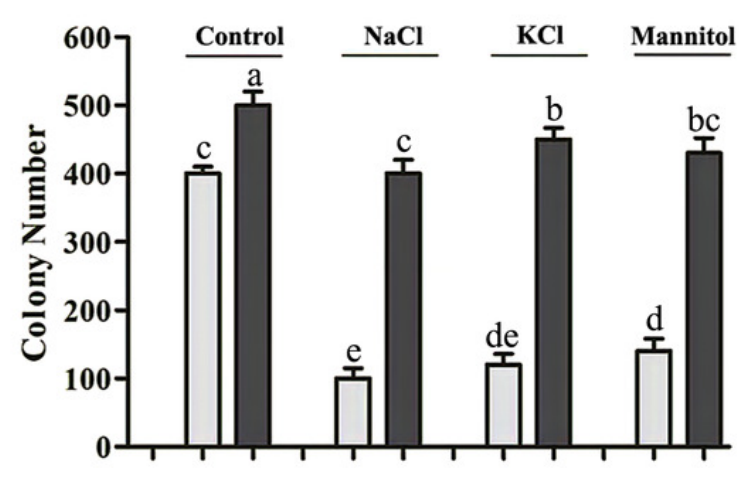

C

CK
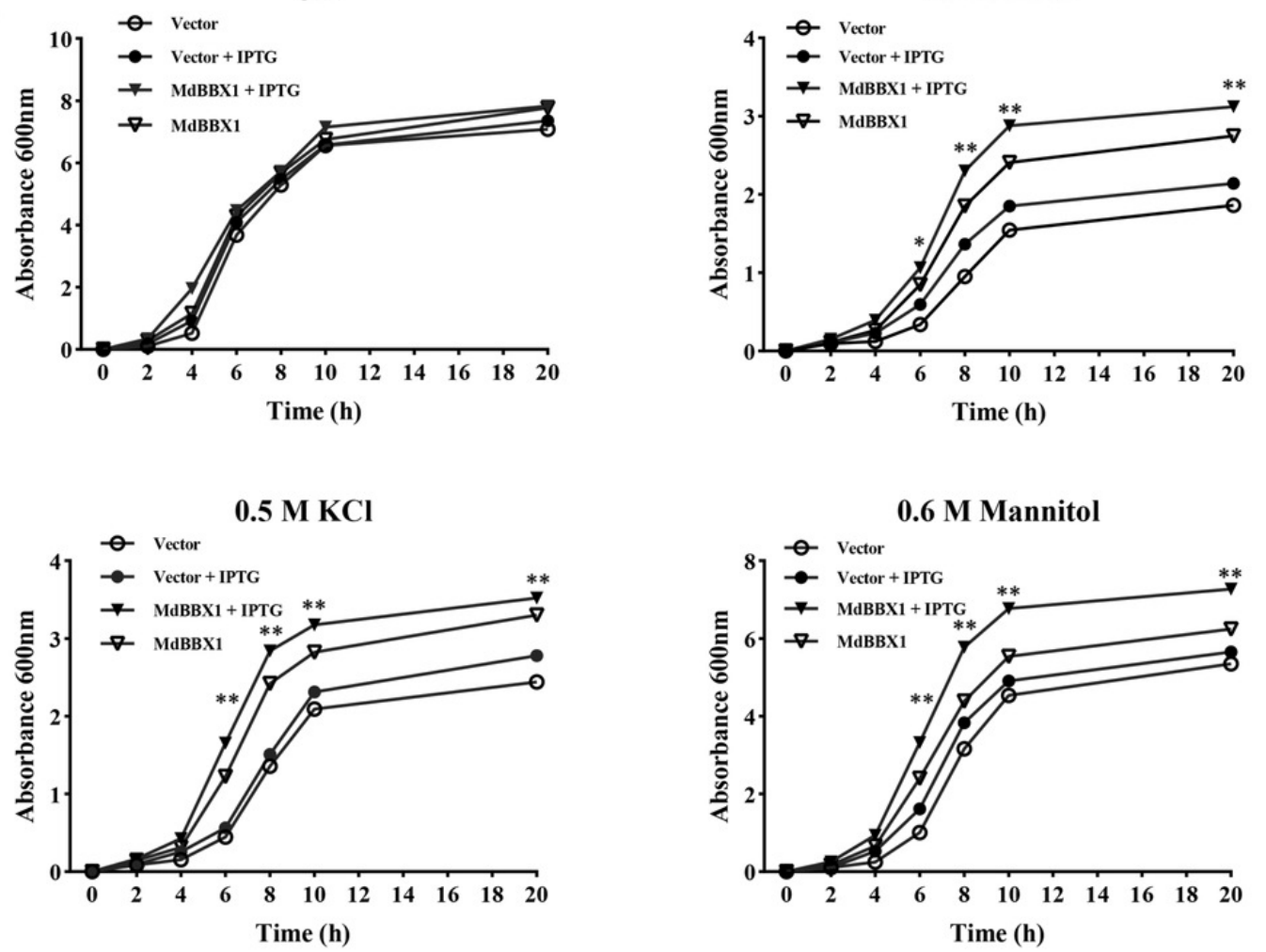


\section{Figure 4}

Germination and root length phenotypes of $M d B B X 1$ overexpression plants under salt tolerance.

(A) Germination phenotype of the wild type (WT) and MdBBXI -overexpressed (OE) lines under $1 / 2 \mathrm{MS}$ medium containing $\mathrm{NaCl}(0,150$ and $200 \mathrm{mM}$ ). Three independent experiments were conducted and each phenotype included 50 seeds. The mean expression value was calculated from three independent replicates. Vertical bars indicate the standard error of mean, $* * P<0.01$ and $* P<0.05$ compared with WT. (B) The root length of WT and MdBBX1transgenic lines in $1 / 2 \mathrm{MS}$ medium containing $150 \mathrm{mM} \mathrm{NaCl}$. Root growth was measured after $\mathrm{NaCl}$ treatment for14 days. The letters above the columns represent significant differences ( $P$ $<0.05$ ) based on Tukey's multiple test. The letters above the columns represents significant differences $(P<0.05)$ based on Tukey's multiple test. 
A
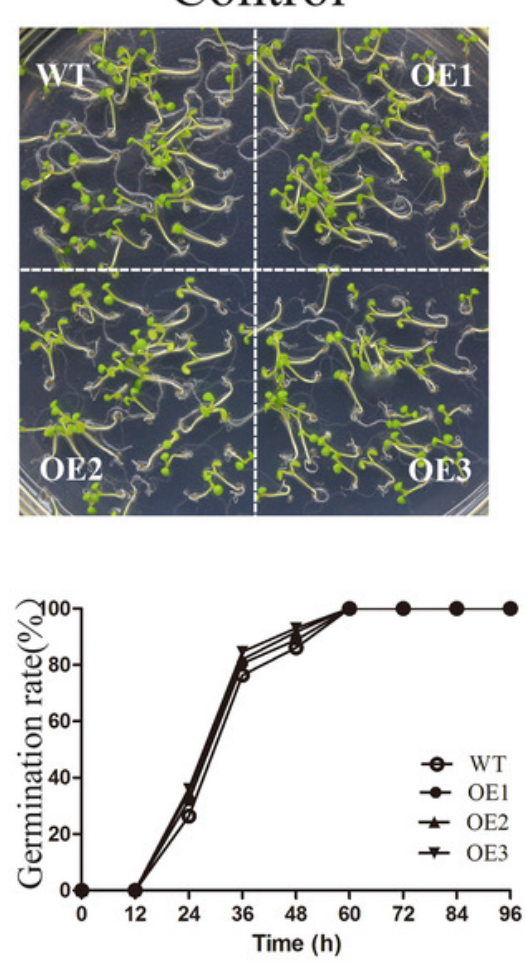

$\mathrm{B}$

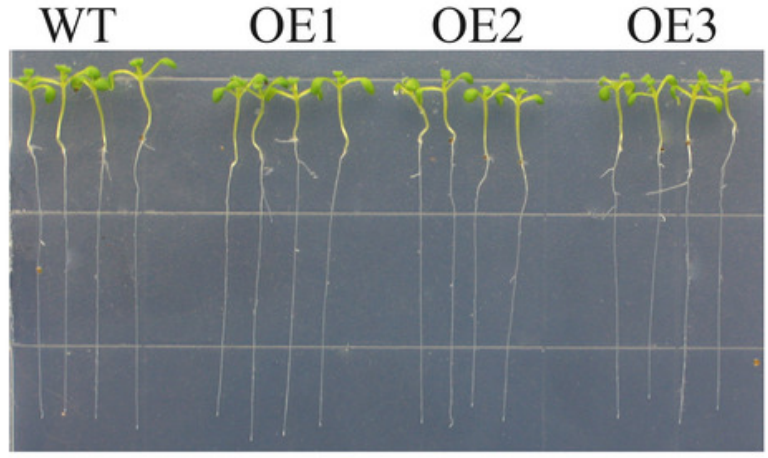

Control

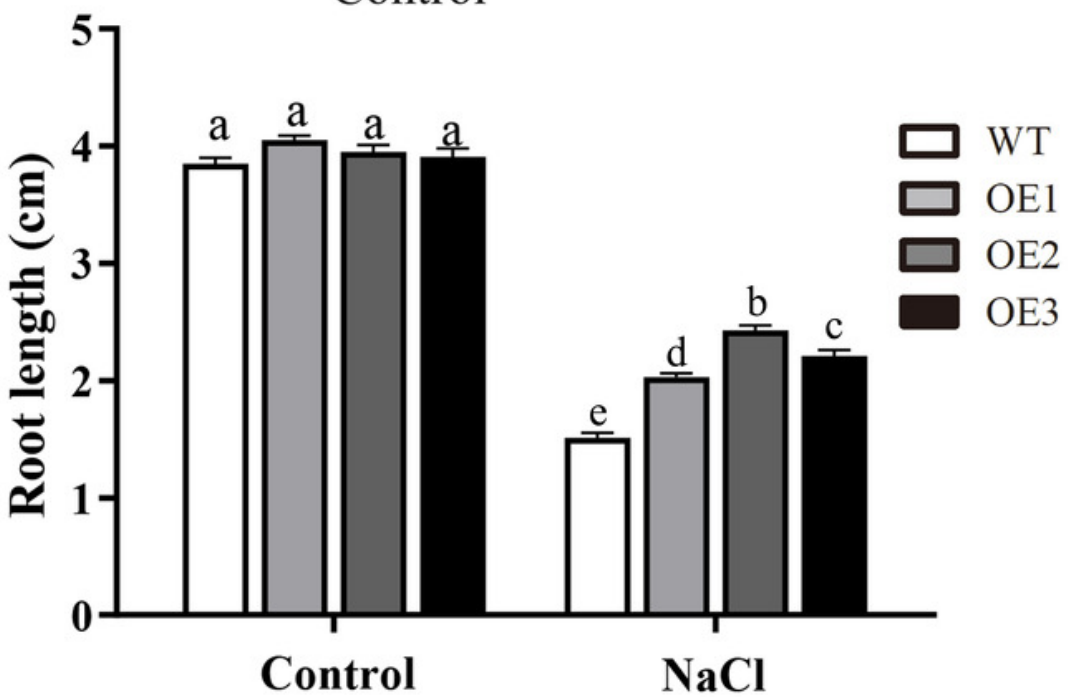

$150 \mathrm{mM} \mathrm{NaCl}$
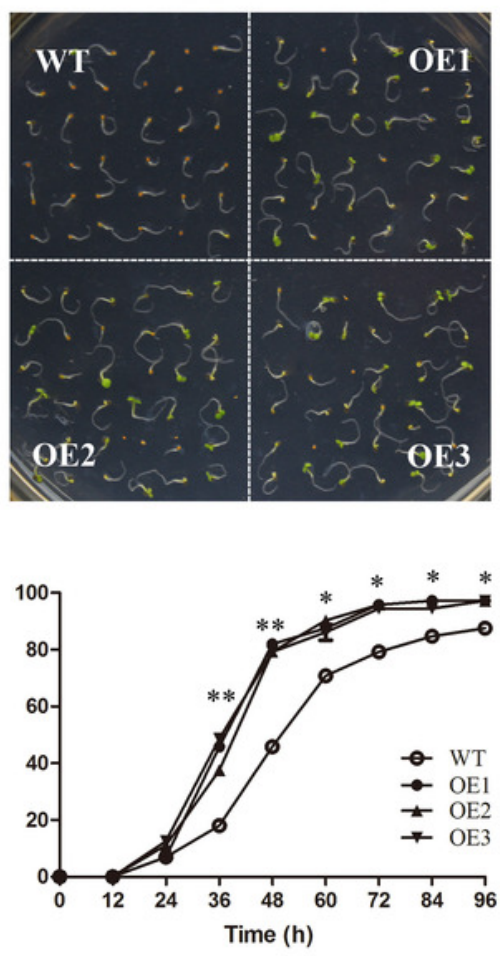

$200 \mathrm{mM} \mathrm{NaCl}$
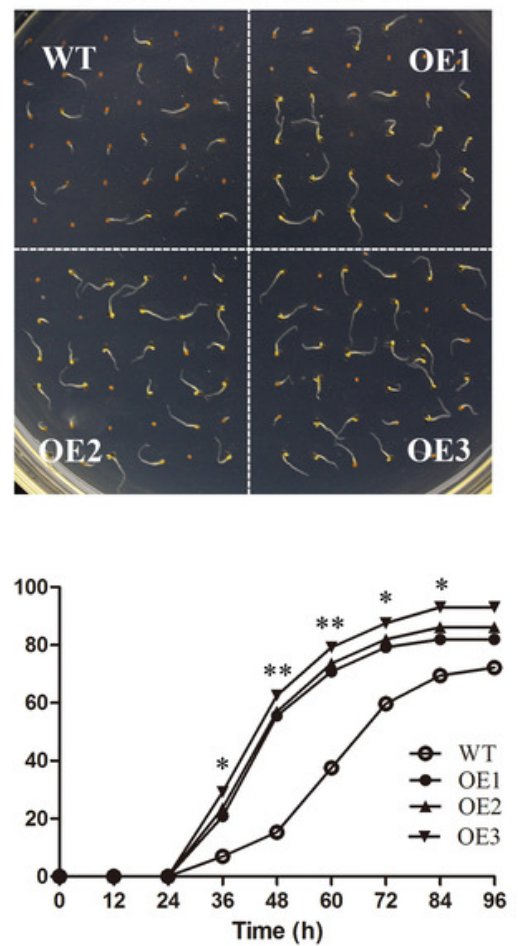

OE1

OE2

OE3

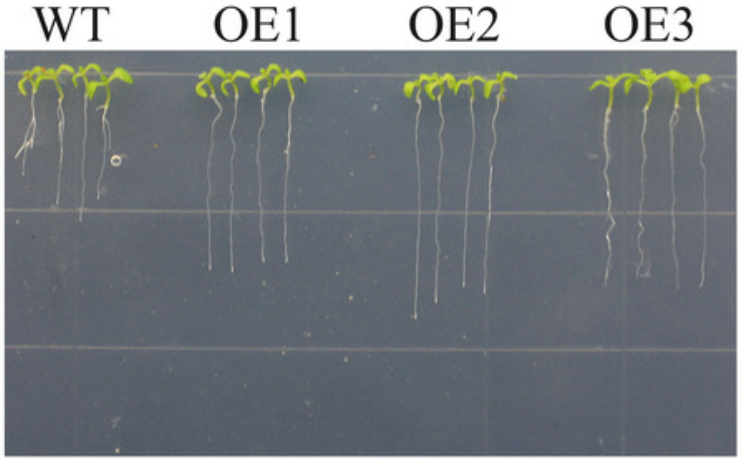

$150 \mathrm{mM} \mathrm{NaCl}$ 


\section{Figure 5}

Overexpression MdBBXI enhanced salt tolerance in transgenic plants.

(A)The representative phenotypes of 3-week old WT and OE seedlings were treated with $\mathrm{NaCl}$ (250 mM) for 3 days to 20 days. (B) Survival rates of WT and transgenic plants after salt stress. PRO (C) and MDA content (D) were measured in WT and transgenic plants after salt stress. Mean values are from three independent replicates and error bars indicate standard deviation. The letters above the columns represent significant differences $(P<0.05)$ based on Tukey's multiple test. 

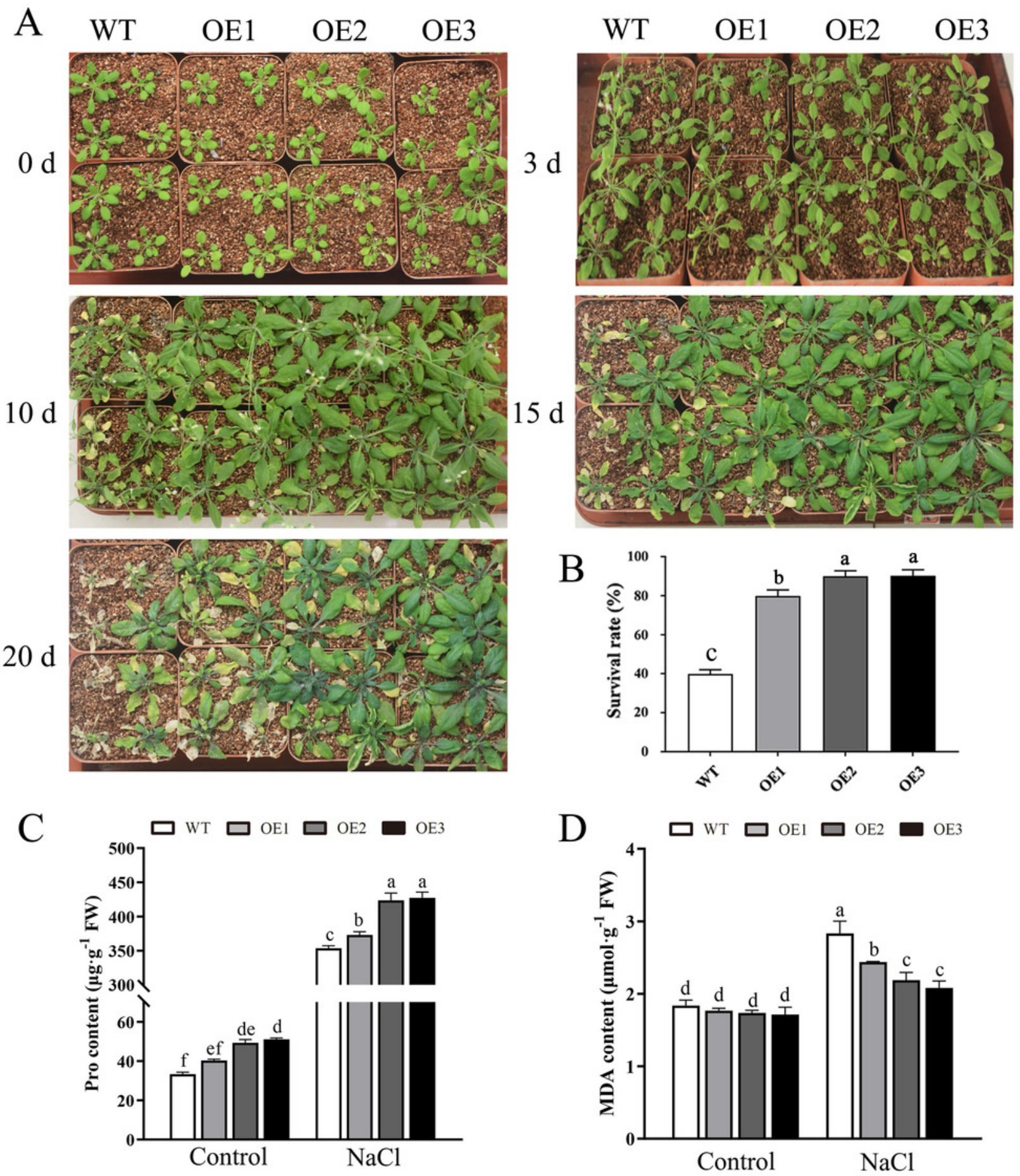


\section{Figure 6}

The phenotype of WT and MdBBX1 transgenic plants in response to drought stress.

(A) The seed germination of WT and the MdBBXI transgenic plants in 1/2 MS medium containing mannitol $(0,300$, or $400 \mathrm{mM})$. Three independent experiments were conducted and each phenotype included 50 seeds. Vertical bars indicate the standard error of mean, ** and * indicate significant differences in comparison with $\mathrm{WT}$ at $\mathrm{P}<0.01$ and $\mathrm{P}<0.05$, respectively. (B) The root length of WT and the transgenic plants of MdBBXI in 1/2 MS medium containing $300 \mathrm{mM}$ mannitol. Root growth of WT and the transgenic plants of $M d B B X 1$ was measured after 14 days. The letters above the columns represent significant differences $(P<0.05)$ based on Tukey's multiple test. (C) The representative phenotypes of WT and OE seedlings were treated with 25\% (w/v) PEG-6000 for 10 days and 25 days. All the columns were represented as mean values of three independent replicates and error bars indicate standard deviation. The letters above the columns represent significant differences $(P<0.05)$ based on Tukey's multiple test. 
A
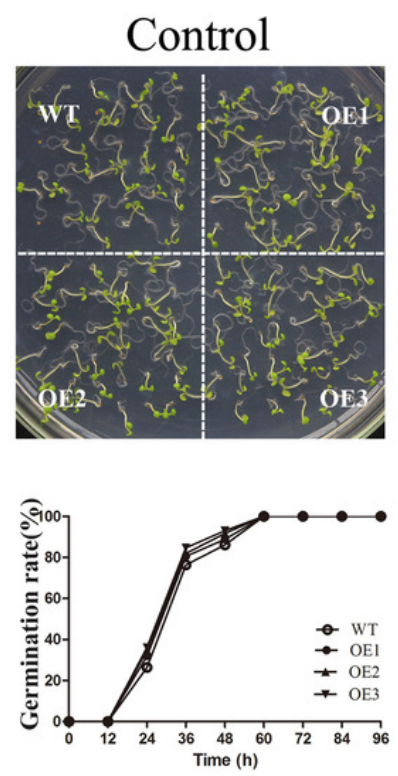
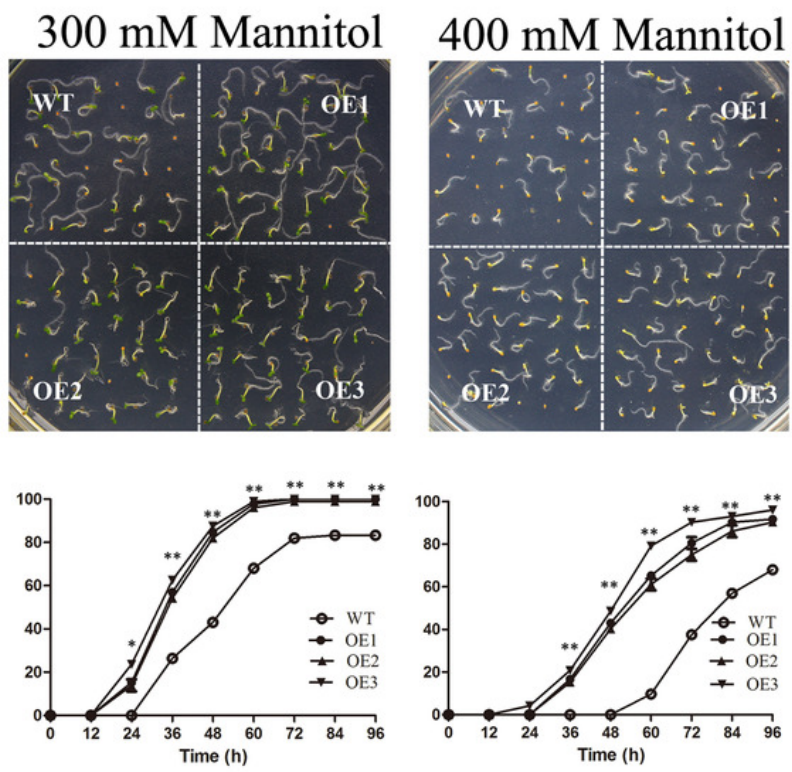

B

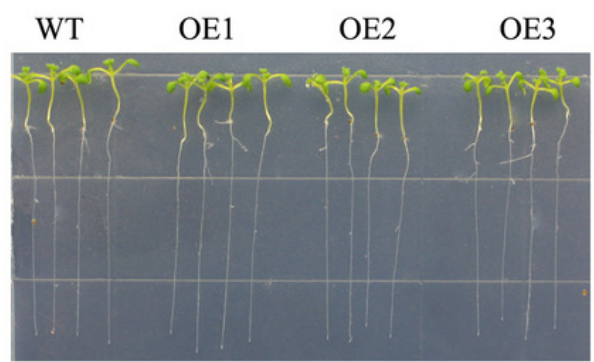

Control

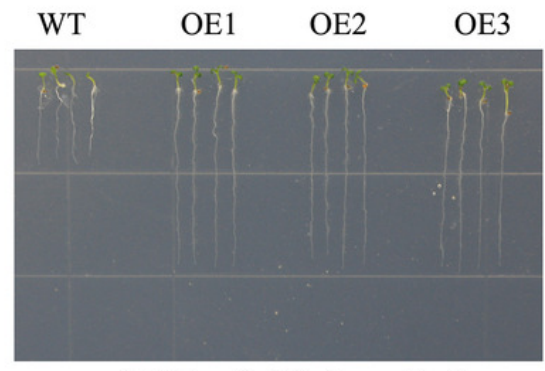

300 mM Mannitol
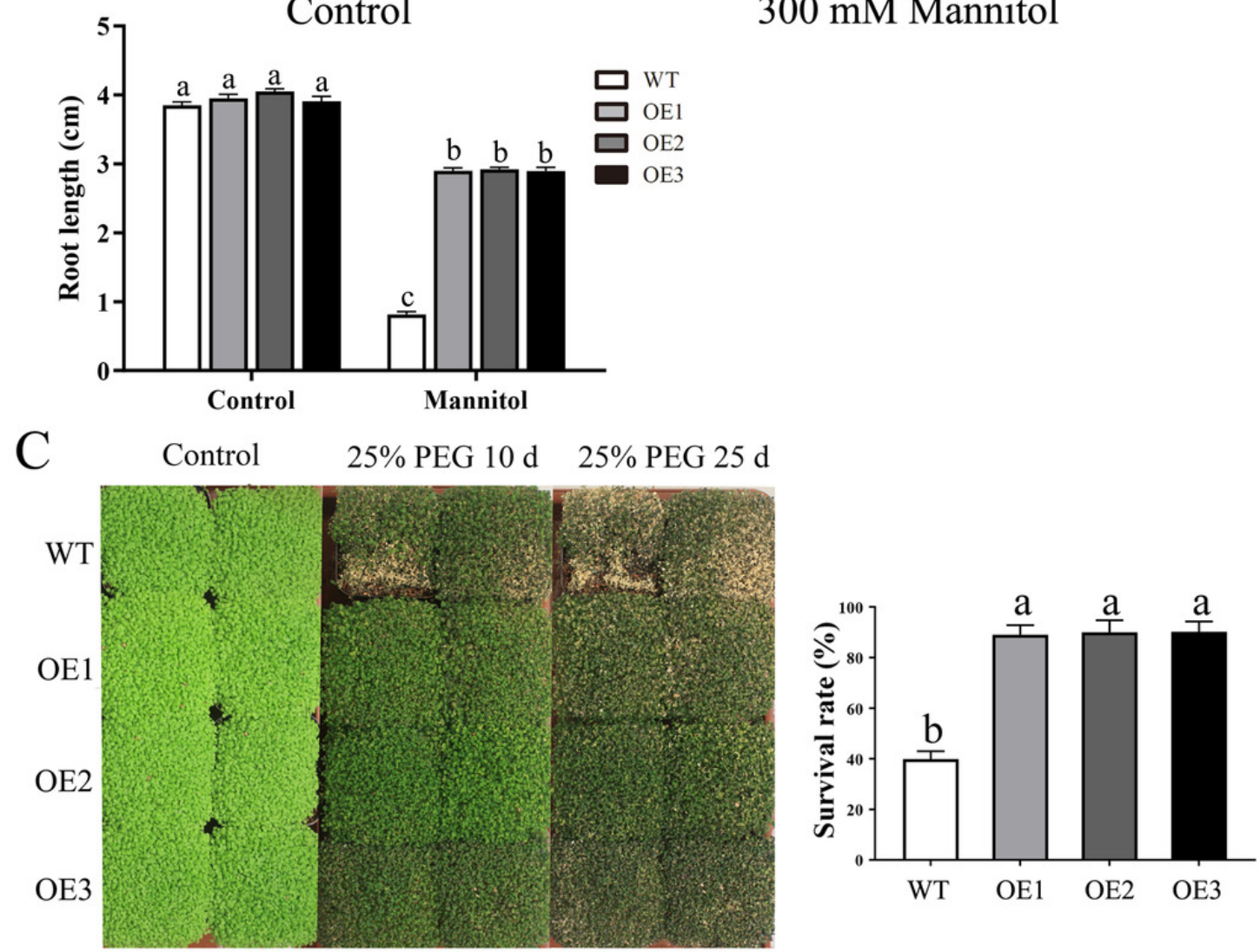


\section{Figure 7}

Germination phenotype of WT and transgenic plants in response to ABA.

(A) Seed germination of WT and OE lines in $1 / 2$ MS medium containing different ABA

concentrations $(0,0.2$ or $0.6 \mu \mathrm{M})$. Three independent experiments were conducted and each phenotype included 50 seeds. Vertical bars indicate the standard error of mean, ** and * indicate significant differences in comparison with $W T$ at $P<0.01$ and $\mathrm{P}<0.05$, respectively. (B) The root length of WT and OE lines in 1/2 MS medium containing $0.6 \mu \mathrm{M}$ ABA. Root growth of WT and OE seedlings was measured after 14 days. (C) The transcriptional levels of HY5 and $A B I 5$ in WT and OE lines after $100 \mu \mathrm{M}$ ABA treatment for $3 \mathrm{~h}$. Little difference was observed among different lines, so one of the OE MdBBX1 transgenic lines was selected as the representative for gene expression analysis. The letters above the columns represent significant differences $(P<0.05)$ based on Tukey's multiple test. 
A
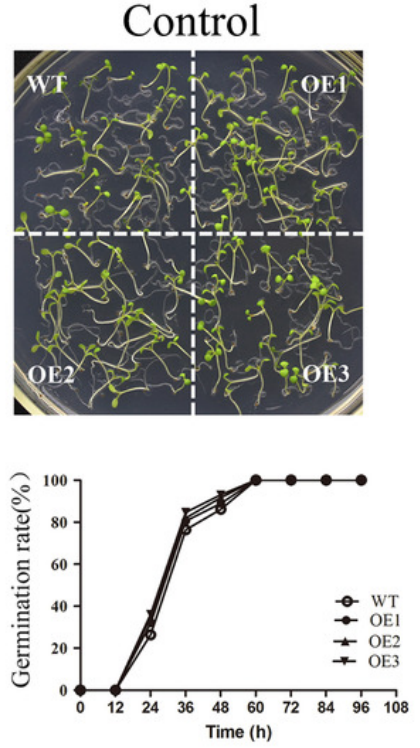

B

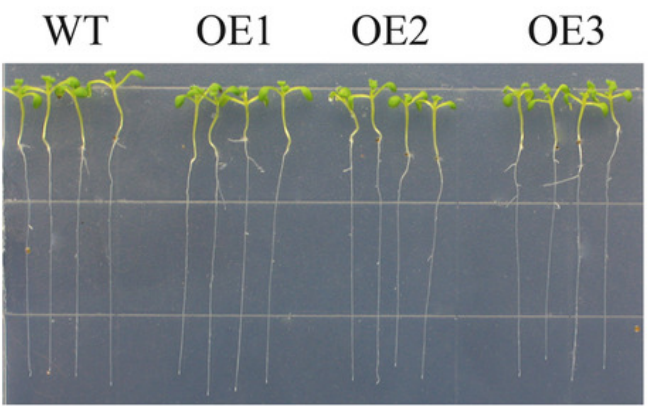

Control
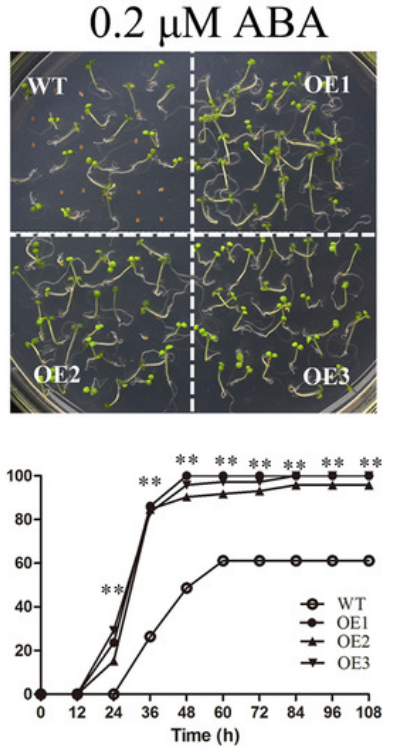
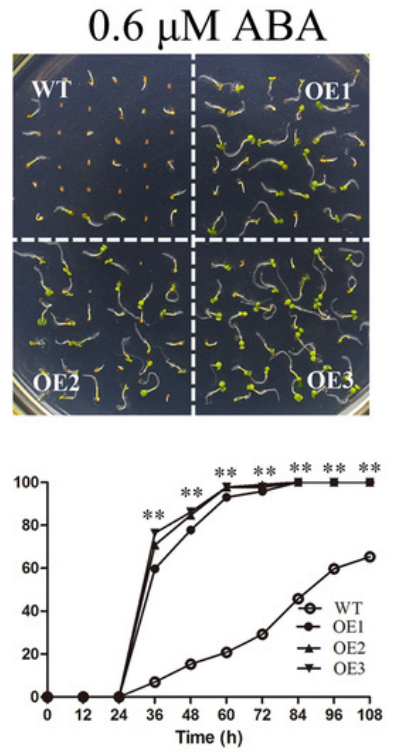

OE1 OE2

OE3

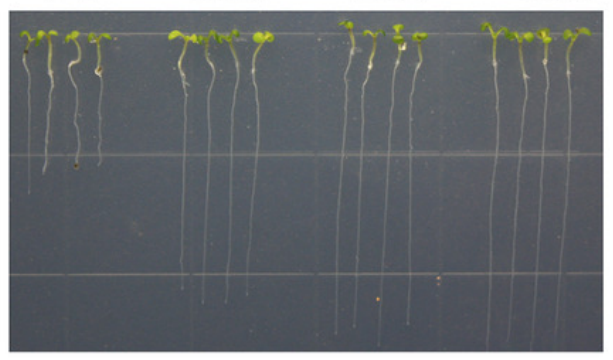

$0.6 \mu \mathrm{M}$ ABA

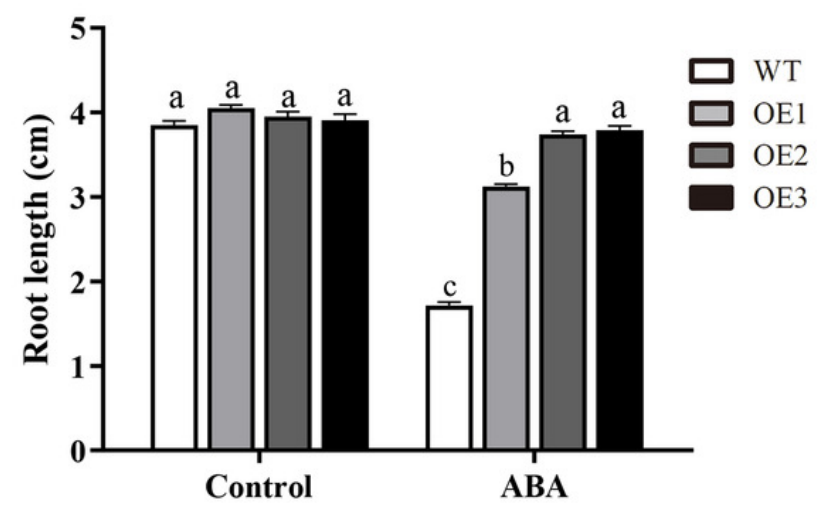

C
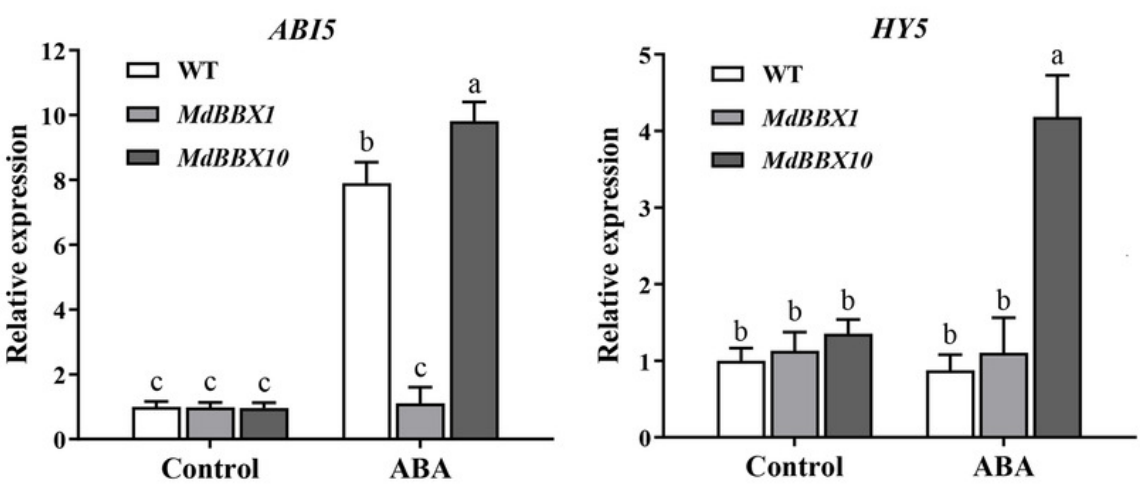


\section{Figure 8}

Analysis of ROS in WT and the MdBBXI transgenic plants after salt and drought stress.

(A) NBT staining of $\mathrm{O}_{2}^{-}$in WT and OE plants after the treatment with $\mathrm{NaCl}$ or PEG-6000 for 6

h. (B) The content of $\mathrm{H}_{2} \mathrm{O}_{2}$ and $\mathrm{O}_{2}{ }^{-}$in WT and transgenic lines after $\mathrm{NaCl}$ treatment. Each column represents the average of three replicates and error bars indicate standard deviation. The letters above the columns represent significant differences $(P<0.05)$ based on Tukey's multiple test.

A

WT

OE1

OE2

OE3

Control

$\mathrm{NaCl}$

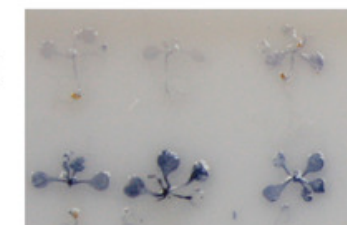

PEG

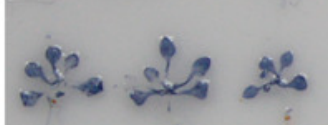

B
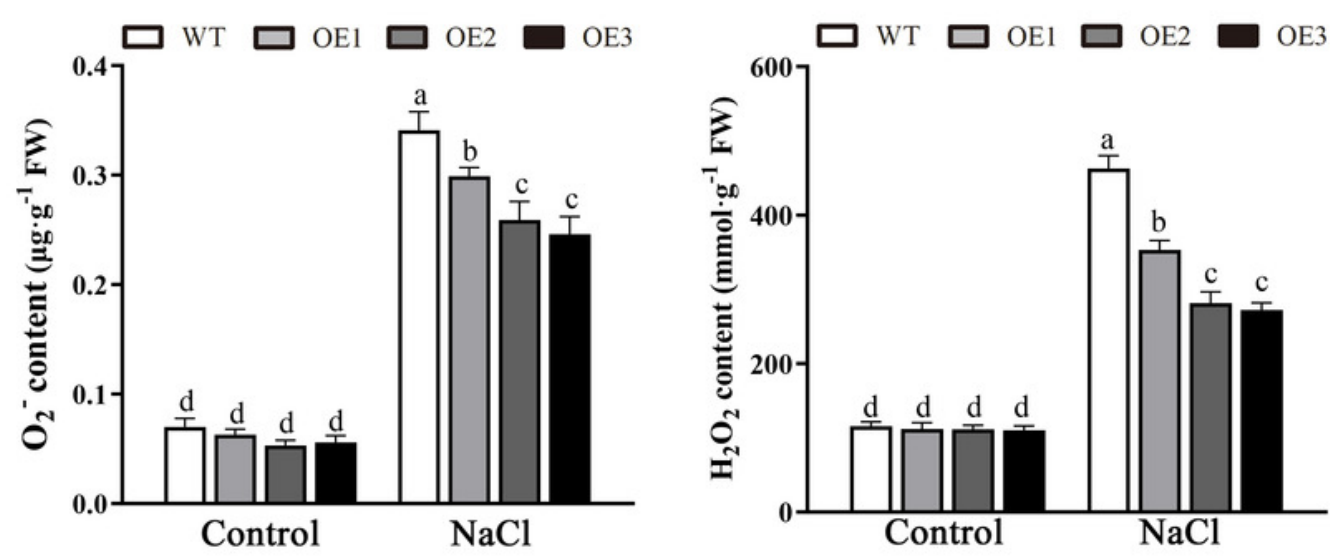


\section{Figure 9}

The activities of ROS-scavenging enzymes in WT and the MdBBX1 transgenic plants after $\mathrm{NaCl}$ treatment.

Each column represents the average of three replicates and error bars indicate standard deviation. The letters above the columns represent significant differences $(P<0.05)$ based on Tukey's multiple test.
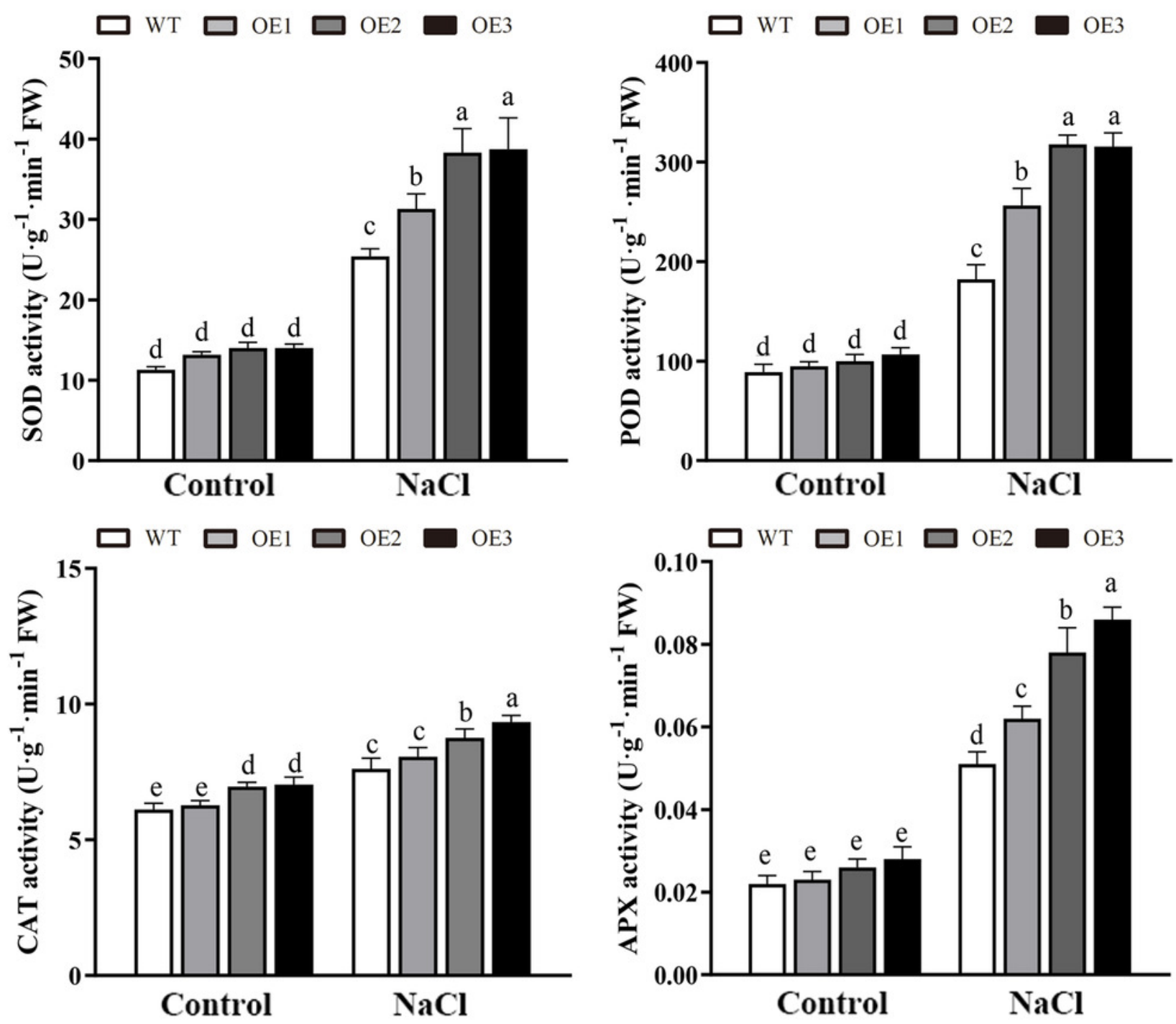